\title{
A damage model of mechanical behavior of porous materials: Application to sandstone
}

\author{
MY $\mathrm{Li}^{1,2}, \mathrm{YJ} \mathrm{Cao}^{2,3}$, WQ Shen ${ }^{2}$ and JF Shao ${ }^{2}$
}

@SAGE

\begin{abstract}
In this work, a multiscale model based on the Fast Fourier Transform (FFT) technique is applied to describe the mechanical behavior of porous materials. The effects of the microstructures (such as pore shape, number, size, distribution and orientation) on the overall strength of the porous medium and its microstress distribution are fully studied. The elastoplastic model is further extended by including a damage process. The influences of microstructure on the damage evolution of the porous medium are discussed and illustrated numerically. Then the proposed multiscale damage model is applied to study the macroscopic behavior of porous sandstone. According to the microstructure of the studied material, a representative elementary volume with randomly distributed spherical pores is considered. The solid phase of the sandstone is assumed to obey the Drucker-Prager criterion. Taking advantage of the FFTbased method, the evolution of generated damage is clearly illustrated during the loading process at the microscopic level. Comparisons between numerical results and experimental data show the efficiency of the proposed numerical model.
\end{abstract}

\section{Keywords}

Homogenization, fast Fourier transform, plasticity, damage, porous material

\section{Introduction}

Porous materials (rocks, chalk, sandstone, cement based materials, etc.) have been widely studied during the last decades for many engineering structures. The pore has a great influence on the permeability and on the material strength. The macroscopic mechanical behavior of a porous material is strongly affected by its porosity which is a main property for the durability analysis. In order to account for a pore's effect, many micromechanical constitutive models have been

\footnotetext{
'State Key Laboratory of Nonlinear Mechanics, Institute of Mechanics, Chinese Academy of Sciences, Beijing 100I90, China ${ }^{2}$ LML, UMR CNRS 8107, University of Lille, France

${ }^{3}$ Geotechnical Research Institute, Hohai University, Nanjing, China

Corresponding author:

WQ Shen, LML, UMR CNRS 8I07, University of Lille, 59655 Villeneuve d'Ascq, France.

Email: Wanqing.Shen@polytech-lille.fr
} 
proposed. Among these models, the most famous and widely used one is proposed by Gurson (1977). In the framework of kinematical limit analysis theory, a macroscopic criterion was delivered in Gurson (1977) for a von Mises material containing a spherical or cylindrical void with a uniform macroscopic strain rate boundary condition. This most widely used criterion takes into account the porosity $f$ of a porous material and improves significantly the phenomenological ones. Based on this pioneering work, a huge number of extensions have been proposed: introducing heuristic parameters to improve the Gurson's criterion (Leblond et al., 1994; Tvergaard, 1981, 1982); changing the incompressible von Mises type matrix to a compressible one (Drucker-Prager type matrix (Guo et al., 2008; Jeong, 2002; Maghous et al., 2009), Mises-Schleicher type matrix (Durban et al., 2010; Lee and Oung, 2000; Monchiet and Kondo, 2012; Shen et al., 2015b), Green type matrix (Shen et al., 2014a,b, 2013, 2012)); considering the void shape from sphere to spheroid (Garajeu and Suquet, 1997; Gologanu et al., 1993, 1994, 1997; Keralavarma and Benzerga, 2010; Monchiet et al., 2014; Pardoen and Hutchinson, 2003; Shen et al., 2011); taking into account the tension-compression asymmetry and the anisotropy of the matrix (Benzerga et al., 1999; Cazacu and Stewart, 2009; Monchiet et al., 2008). On the other hand, some criteria have been established for a porous material with a von Mises type matrix by using variational techniques (Michel and Suquet, 1992; Ponte Castaneda, 1991; Sun and Wang, 1989, etc.). Recently, a quasi-lower bound was proposed in Cheng et al. (2014) with a stress variational homogenization and improved by Shen et al. (2015a) by adopting a fully statically admissible microscopic stress field. Lately, a homogenized Gurson type criterion for porous ductile materials considering both void shape and plastic anisotropy effects was proposed in Morin et al. (2015). The void growth and void coalescence theories have been unified in a yield criterion in Morin et al. (2016). The Mohr-Coulomb type matrix was studied in Anoukou et al. (2016) by using a kinematic limit analysis approach for a ductile porous medium.

In most of the above-mentioned models, different kinds of homogenization techniques have been used. As it is generally difficult to exactly consider local fields of stress, strain and damage at a microscopic scale, simplifications and assumptions are therefore needed. For instance, in order to establish a macroscopic criterion, a unit-cell is usually necessary to present the studied porous material. For example, a hollow sphere subjected to a uniform macroscopic strain rate boundary condition was studied in the famous Gurson's model. For a porous medium with spheroidal voids, a spheroidal volume containing a confocal spheroidal void is considered. With the homogenization procedure, the effect of porosity on the effective yield surface of porous materials can be explicitly taken into account, but the influences of interactions between voids and other microstructure information (void shapes, sizes, orientation and distribution, etc.) are not so easily considered simultaneously in a criterion. A numerical study is an efficient way to solve this problem. Concerning the three-dimensional computational homogenization for a porous medium with multiple voids, the effective behavior of ductile metals containing spherical voids was recently studied in Fritzen et al. (2012) on a computational basis. According to the computational results, the GursonTvergaard-Needleman (GTN) model was extended. The influence of pore clustering on the macroscopic material behavior has been investigated in Bilger et al. (2005) by using the Fast Fourier Transform (FFT). Based on works of Moulinec and Suquet (1994, 1998), the FFT method is considered as an alternative approach to the finite element method (FEM) to compute the effective properties of composite materials with a periodic microstructure. Different from the FEM, this method does not require the process of meshing. By discretizing the studied heterogeneous material into a series of grid points, the FFT approach is able to efficiently describe non-regular geometrical forms of complex microstructure. Different mechanical properties can be assigned to each point according to its location inside the heterogeneous microstructure. This approach was further 
improved by some accelerated schemes to improve its computational efficiency (Eyre and Milton, 1999; Michel et al., 1999, 2000, 2001; Monchiet and Bonnet, 2012).

However, most of the previous works on FFT simulations have been essentially devoted to metal or composite materials under tensile loading. The solid phase was described by a pressure-independent criterion. Furthermore, porous materials with a single porosity have been generally investigated. The objective of the present work is to provide a new numerical micromechanical model based on the FFT method to describe effective nonlinear behaviors of porous geomaterials under compression-dominated stresses. Both plastic deformation and induced damage will be considered. The plastic behavior of the solid phase is described by a pressure-sensitive non-associated plastic model. Particular attention will be paid to the progressive damage process in porous materials with different pore distributions. The paper is organized as follows. The FFT-based numerical method is firstly recalled in section "FFT-based numerical method". Taking advantage of this method without meshing, the effects of microstructure (with different void number, shape, size and orientation) on the macroscopic behavior of a porous medium are extensively studied in section "Influence of microstructure on the mechanical behavior of porous material". Then in section "Extension to a damage model", the emphasis is put on the description of the damage process in porous materials. In particular, the influences of microstructure on the damage evolution are studied. In section "Application to the sandstone with a simple damage criterion", the proposed plastic-damage model is applied to describe the mechanical behavior of a typical porous sandstone.

\section{FFT-based numerical method}

\section{General framework of FFT technique}

In this section, the FFT technique will be firstly recalled based on the works of Moulinec and Suquet $(1994,1998)$. For the sake of clarity, we consider an inhomogeneous linear elastic material. The unit cell of the material is subjected to a uniform macroscopic strain $\boldsymbol{E}$ on its boundary. Due to the material heterogeneity, the local stress and strain fields are not uniform. The non-uniform strain field is defined by a periodic fluctuation displacement field $\boldsymbol{u}^{*}(\boldsymbol{x})$. Therefore, the local strain field inside the unit cell, $\boldsymbol{\varepsilon}(\mathbf{u}(\mathbf{x}))$, can be expressed by

$$
\varepsilon(\boldsymbol{u}(\boldsymbol{x}))=\varepsilon\left(u^{*}(x)\right)+E
$$

with the conditions $\langle\boldsymbol{\varepsilon}\rangle=\boldsymbol{E}$ and $\left\langle\boldsymbol{\varepsilon}\left(\boldsymbol{u}^{*}(\boldsymbol{x})\right)\right\rangle=0$, and the operator $<\cdot>$ denoting the volumetric averaging on the unit cell.

The problem to be solved here is to determine the local stress field using the local constitutive relations and then to evaluate the macroscopic stress by making the volumetric average over the unit cell, as the macroscopic response to the prescribed macroscopic strain. To this end, denote $\mathbb{C}(\boldsymbol{x})$ as the non-uniform local elastic stiffness tensor. The local governing equations are given by

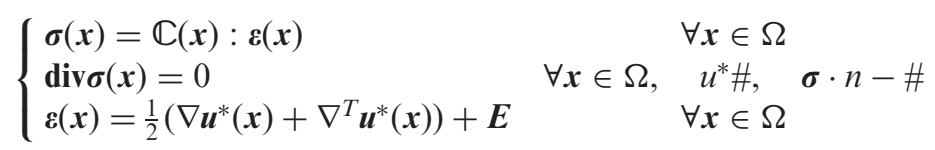

In these relations, the symbol \# denotes the periodic condition while -\# the anti-periodic one.

By introducing a homogeneous elastic reference material with a constant stiffness tensor $\mathbb{C}^{0}$ and after defining a polarization tensor field $\tau(\boldsymbol{x})=\left(\mathbb{C}(\boldsymbol{x})-\mathbb{C}^{0}\right): \boldsymbol{\varepsilon}(\boldsymbol{x})$, the above local problem can be 
reduced to the periodic Lippman-Schwinger problem (Moulinec and Suquet, 1998). The solution of this problem in the real space can be determined by using the periodic Green operator $\Gamma^{0}(\boldsymbol{x})$. One gets

$$
\varepsilon(x)=-\Gamma^{0}(x) * \tau(x)+E
$$

The convolution operator in the real space "*" is difficult to calculate but it can be reduced to a simple product operator in the Fourier space. Indeed, the expression of the Green operator in the Fourier space is explicitly known. Therefore after making the FFT, the solution to the local problem can be easily expressed by

$$
\hat{\boldsymbol{\varepsilon}}(\xi)=-\hat{\Gamma}^{0}(\xi): \hat{\tau}(\xi) \quad \forall \xi \neq \boldsymbol{0}, \quad \hat{\boldsymbol{\varepsilon}}(\boldsymbol{O})=\boldsymbol{E}
$$

In this solution, $\hat{\tau}$ and $\hat{\Gamma}^{0}$ are the polarization tensor and periodic Green operator in Fourier space respectively. Further, the constant stiffness tensor $\mathbb{C}^{0}$ of the isotropic elastic reference material can be expressed in terms of the two Lamé coefficients $\lambda^{0}$ and $\mu^{0}$. The Green operator in the Fourier space can be explicitly given by

$$
\hat{\Gamma}_{i j k l}(\xi)=\frac{1}{4 \mu^{0}|\xi|^{2}}\left(\delta_{i k} \xi_{j} \xi_{l}+\delta_{j k} \xi_{i} \xi_{l}+\delta_{i l} \xi_{j} \xi_{k}+\delta_{j l} \xi_{i} \xi_{k}\right)-\frac{\lambda^{0}+\mu^{0}}{\mu^{0}\left(\lambda^{0}+2 \mu^{0}\right)} \frac{\xi_{i} \xi_{j} \xi_{k} \xi_{l}}{|\xi|^{4}}
$$

Therefore, the FFT-based numerical method consists of finding an appropriate non-uniform strain field and the corresponding stress field, which verify the local constitutive relations, equilibrium equations and boundary conditions on the unit cell. To do this, an iterative algorithm is needed. By making use of the Green operator property $\Gamma^{0} *\left(\mathbb{C}^{0}: \boldsymbol{\varepsilon}\right)=\boldsymbol{\varepsilon}$, and based on the previous works by (Moulinec and Suquet, 1994, 1998), the following iterative algorithm is adopted

$$
\begin{cases}\text { Initialization }: & \boldsymbol{\varepsilon}^{0}(\boldsymbol{x})=\boldsymbol{E} \quad \forall \boldsymbol{x} \in \Omega \\ & \boldsymbol{\sigma}^{0}(\boldsymbol{x})=\mathbb{C}(\boldsymbol{x}): \boldsymbol{\varepsilon}^{0}(\boldsymbol{x}) \quad \forall \boldsymbol{x} \in \Omega \\ \text { Iterate } i+1 & \text { the previous } \boldsymbol{\varepsilon}^{i} \text { and } \boldsymbol{\sigma}^{i} \text { being known at each position } \boldsymbol{x} \\ (\mathrm{a}) & \hat{\boldsymbol{\sigma}}^{i}=\mathcal{F}\left(\boldsymbol{\sigma}^{i}\right) \\ (\mathrm{b}) & \text { Verify the convergence and update the stress/strain } \\ (\mathrm{c}) & \hat{\boldsymbol{\varepsilon}}^{i+1}(\boldsymbol{\xi})=\hat{\boldsymbol{\varepsilon}}^{i}(\boldsymbol{\xi})-\hat{\Gamma}^{0}(\boldsymbol{\xi}): \hat{\boldsymbol{\sigma}}^{i}(\boldsymbol{\xi}) \quad \forall \boldsymbol{\xi} \neq \boldsymbol{0}, \quad \hat{\boldsymbol{\varepsilon}}^{i+1}(\boldsymbol{0})=\boldsymbol{E} \\ (\mathrm{d}) & \boldsymbol{\varepsilon}^{i+1}=\mathcal{F}^{-1}\left(\hat{\boldsymbol{\varepsilon}}^{i+1}\right) \\ \text { (e) } & \boldsymbol{\sigma}^{i+1}(\boldsymbol{x})=\mathbb{C}(\boldsymbol{x}): \boldsymbol{\varepsilon}^{i+1}(\boldsymbol{x}) \quad \forall \boldsymbol{x} \in \Omega\end{cases}
$$

In these relations, $\boldsymbol{x}$ and $\boldsymbol{\xi}$ denote the coordinates in real space and Fourier space, respectively. The symbols $\mathcal{F}$ and $\mathcal{F}^{-1}$ represent correspondingly the FFT and inverse FFT operators. The convergence condition is controlled by the equilibrium equation and performed in Fourier space to avoid the difficulty of the computation for the differential operator

$$
\operatorname{err}^{i}=\frac{\left(<\left\|\operatorname{div} \boldsymbol{\sigma}^{i}(\boldsymbol{x})\right\|^{2}>\right)^{1 / 2}}{\left\|<\boldsymbol{\sigma}^{i}(\boldsymbol{x})>\right\|}=\frac{<\left\|\boldsymbol{\xi} \cdot \hat{\boldsymbol{\sigma}}^{i}(\boldsymbol{\xi})\right\|^{2}>^{1 / 2}}{\left\|\hat{\sigma}^{i}(\boldsymbol{0})\right\|} \leq \omega
$$

and the matrix 2-norm is utilized here as the norm of the second-order tensor. $\omega$ is a chosen value of convergence tolerance. 
The choice of the reference stiffness tensor $\mathbb{C}^{0}$ can significantly affect the rate of convergence. In practice, according to Moulinec and Suquet (1998), the best rate of convergence is provided with the following values of Lamé coefficients $\lambda^{0}$ and $\mu^{0}$ for the reference material

$$
\begin{aligned}
& \lambda^{0}=\frac{1}{2}\left(\inf _{\boldsymbol{x} \in V} \lambda(\boldsymbol{x})+\sup _{\boldsymbol{x} \in V} \lambda(\boldsymbol{x})\right) \\
& \mu^{0}=\frac{1}{2}\left(\inf _{\boldsymbol{x} \in V} \mu(\boldsymbol{x})+\sup _{\boldsymbol{x} \in V} \mu(\boldsymbol{x})\right)
\end{aligned}
$$

\section{Discrete FFT model}

In the above FFT-based numerical model, a continuous polarization tensor field should be determined. In practice, in view of its numerical implementation, a discrete FFT-based model is defined (Moulinec and Suquet, 1994, 1998). We shall determine a discrete distribution of the polarization tensor at a limited number of points. Therefore, the unit cell is discretized by a regular grid composed of a limited number of points in each direction in real space. For example, this discrete grid is composed of $N 1 \times N 2 \times N 3$ voxels in the three-dimensional case. The coordinates of voxels in real space are denoted by $\boldsymbol{x}_{p}\left(i_{1}, i_{2}, i_{3}\right)$ which are linked to the coordinates in Fourier space, $\boldsymbol{\xi}\left(i_{1}, i_{2}, i_{3}\right)$. The number of points in each direction depends on the choice of resolution. The relationships between the two coordinates and the grid information are given by

$$
\boldsymbol{x}_{p}\left(i_{i}, i_{2}, i_{3}\right)=i_{k} \cdot \frac{T_{k}}{N_{k}}, \quad i_{k}=0,1, \ldots, N_{k-1}, \quad k=1,2,3
$$

The coordinates of $\xi\left(i_{1}, i_{2}, i_{3}\right)$ for the case of $N_{k}$ is even

$$
\xi\left(i_{1}, i_{2}, i_{3}\right)=\left(i_{k}-\frac{N_{k}-1}{2}\right) \frac{1}{T_{k}}
$$

and the case of $N_{k}$ is odd

$$
\xi\left(i_{1}, i_{2}, i_{3}\right)=\left(i_{k}-\frac{N_{k}}{2}+1\right) \frac{1}{T_{k}}
$$

where $T_{k}$ is the period of the model in the $k$ th direction $(k=1,2,3)$.

Therefore, the iterative numerical algorithm presented above will be applied to each discrete point $\boldsymbol{x}_{\boldsymbol{p}}$ in real space or $\boldsymbol{\xi}_{\boldsymbol{p}}$ in Fourier space.

\section{Application to nonlinear heterogeneous materials}

The above numerical method for a linear inhomogeneous material is now extended to nonlinear heterogeneous materials. As indicated in Moulinec and Suquet (1994, 1998) and Jiang and Shao (2012), it is generally convenient to express nonlinear constitutive relations in an incremental form. For this purpose, the total loading path is divided into a limited number of steps $N$. Starting from the initial conditions, at the end of step $n$, the local fields of stress, strain and internal variables $\left(\left(\boldsymbol{\sigma}^{n}, \boldsymbol{\varepsilon}^{n}, \boldsymbol{V}^{n}\right)\right)$ are all known. Considering now the loading step $n+1$, an increment of macroscopic strain $\Delta \boldsymbol{E}^{n+1}$ is applied to the unit cell. The problem to be solved is to find the corresponding macroscopic stress increment $\Delta^{n+1}$ by using nonlinear local constitutive relations. 
To this end, the iterative algorithm presented above is now applied to each loading step. The time-independent behavior is only considered here. Without losing the generality, the local nonlinear constitutive relations can be expressed in the following incremental form

$$
\Delta \sigma(x)=\mathbb{C}^{\tan }(\boldsymbol{x}): \Delta \varepsilon(\boldsymbol{x})
$$

The fourth-order tensor $\mathbb{C}^{\tan }(\boldsymbol{x})$ denotes the tangent operator which depends on the loading history and loading direction.

Accordingly, the FFT-based iterative numerical algorithm for nonlinear heterogeneous materials at the loading step $n+1$ is defined by (for the sake of simplicity, the step index $(n+1)$ in omitted in the iteration loop)

$\begin{cases}\text { Initialization : } & \boldsymbol{\varepsilon}^{(n+1) 0}\left(\boldsymbol{x}_{\boldsymbol{p}}\right)=\boldsymbol{\varepsilon}^{n}\left(\boldsymbol{x}_{\boldsymbol{p}}\right)+\Delta \boldsymbol{E}^{n+1} \quad \forall \boldsymbol{x}_{p} \in \Omega, \\ & \text { Call the elastoplastic subroutine to calculate } \mathbb{C}^{\tan (n+1) 0}\left(\boldsymbol{x}_{\boldsymbol{p}}, \boldsymbol{V}^{(n+1) 0}\right) \\ & \boldsymbol{\sigma}^{(n+1) 0}\left(\boldsymbol{x}_{p}\right)=\boldsymbol{\sigma}^{n}\left(\boldsymbol{x}_{p}\right)+\mathbb{C}^{\tan (n+1) 0}\left(\boldsymbol{x}_{\boldsymbol{p}}\right): \Delta \boldsymbol{\varepsilon}^{(n+1) 0}\left(\boldsymbol{x}_{\boldsymbol{p}}\right) \quad \forall \boldsymbol{x}_{p} \in \Omega \\ \text { Iterate } i+1 \quad & \text { The previous } \boldsymbol{\varepsilon}^{i} \text { and } \boldsymbol{\sigma}^{i} \text { are known at each position } \boldsymbol{x}_{p} \\ (\text { a) } & \hat{\boldsymbol{\sigma}}^{i}=\mathcal{F}\left(\boldsymbol{\sigma}^{i}\right) \\ \text { (b) } & \text { Verify the convergence and updatethe stress } / \text { strain } \\ \text { (c) } & \hat{\boldsymbol{\varepsilon}}^{i+1}\left(\boldsymbol{\xi}_{p}\right)=\hat{\boldsymbol{\varepsilon}}^{i}\left(\boldsymbol{\xi}_{p}\right)-\hat{\Gamma}^{0}\left(\boldsymbol{\xi}_{p}\right): \hat{\boldsymbol{\sigma}}^{i}\left(\boldsymbol{\xi}_{p}\right) \quad \forall \boldsymbol{\xi}_{p} \neq \boldsymbol{0}, \quad \hat{\boldsymbol{\varepsilon}}^{i+1}(\boldsymbol{\theta})=\boldsymbol{E}^{(n+1)} \\ \text { (d) } & \boldsymbol{\varepsilon}^{i+1}=\mathcal{F}^{-1}\left(\hat{\boldsymbol{\varepsilon}}^{i+1}\right) \\ \text { (e) } & \text { Call the elastoplastic subroutine to calculate } \mathbb{C}^{\tan (i+1)}\left(\boldsymbol{x}_{\boldsymbol{p}}, \boldsymbol{V}^{(i+1)}\right) \\ \text { (f) } & \boldsymbol{\sigma}^{i+1}\left(\boldsymbol{x}_{p}\right)=\boldsymbol{\sigma}^{n}\left(\boldsymbol{x}_{p}\right)+\mathbb{C}^{\tan (i+1)}\left(\boldsymbol{x}_{\boldsymbol{p}}\right): \Delta \boldsymbol{\varepsilon}^{i+1}\left(\boldsymbol{x}_{\boldsymbol{p}}\right) \quad \forall \boldsymbol{x}_{p} \in \Omega \\ \text { (g) } & \text { Conserve } \boldsymbol{V}^{i+1}\end{cases}$

\section{Comparison with the FEM solution}

For the purpose of validation, the accuracy of the FFT-based method is firstly verified by comparing with reference solutions obtained from finite element simulations. A simplified porous medium is considered with a porosity $f=10 \%$ : a cubic unit cell containing a centered spherical pore (Figure 1). These two methods adopt totally different strategies to discretize the structure. The FEM method meshes the structure with a certain shape of element (37,040 hexagonal elements in Figure 1(a)). On the contrary, the FFT-based method discretizes the structure with regular voxels $(128 \times 128 \times 128$ voxels in Figure 1(b)).

The solid matrix in Figure 1(a) and 1(b) is characterized by a von Mises criterion with an isotropic plastic hardening

$$
f=\sigma_{\mathrm{eq}}-\left(\sigma_{0}+H \gamma^{m}\right)=0
$$

where $\sigma_{\mathrm{eq}}$ indicated the equivalent stress and computed as $\sigma_{\mathrm{eq}}=\left(\frac{3}{2} s: s\right)^{1 / 2} . s$ is the deviatoric part of the stress $\boldsymbol{\sigma}$. $H$ and $m$ are two plastic hardening parameters. The plastic variable $\gamma$ is determined by an associated plastic flow rule

$$
\dot{\boldsymbol{\varepsilon}} p=\dot{\lambda} \frac{\partial f}{\partial \boldsymbol{\sigma}}=\frac{3}{2} \dot{\gamma} \frac{\boldsymbol{s}}{\sigma_{\mathrm{eq}}}, \quad \dot{\gamma}=\dot{\lambda}
$$


(a)

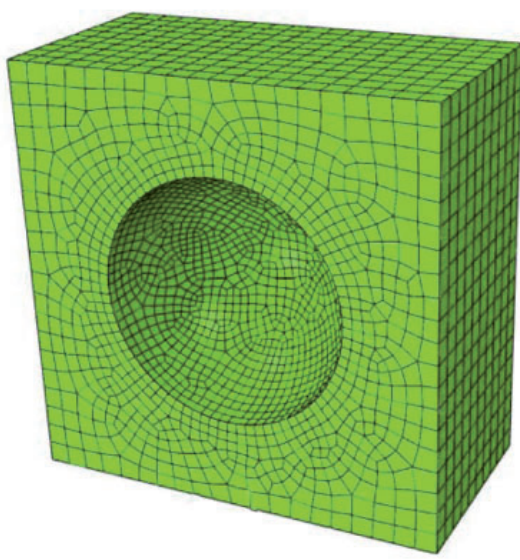

(b)

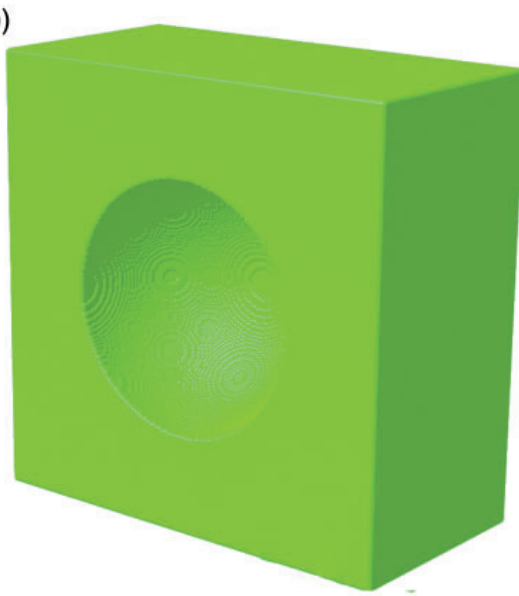

Figure I. Microstructure with a centered spherical void: porosity $f=10 \%$. (a) FEM mesh with 37040 hexagonal elements. (b) FFT discretization with regular $128 \times 128 \times 128$ voxels.

Table I. Elastic and plastic parameters of the solid matrix.

\begin{tabular}{lllll}
\hline$E(\mathrm{GPa})$ & $v$ & $\sigma_{0}(\mathrm{MPa})$ & $H(\mathrm{MPa})$ & $m$ \\
\hline 10.0 & 0.25 & 45.0 & 150.0 & 0.5 \\
\hline
\end{tabular}

Table 2. Numerical comparison between FFT method and finite element solution.

\begin{tabular}{|c|c|c|c|c|c|c|c|c|c|}
\hline \multicolumn{5}{|l|}{ FFT } & \multicolumn{5}{|l|}{ FEM } \\
\hline Resolution (N) & Points & CPU time (s) & $\sigma^{P}(\mathrm{MPa})$ & Error (\%) & Mesh & Nodes & CPU time (s) & $\sigma^{P}(\mathrm{MPa})$ & Error (\%) \\
\hline 8 & 512 & 388.2 & 63.1 & 1.54 & 1 & 1920 & 409.1 & 71.1 & 10.92 \\
\hline 16 & 4096 & $134 \mid .8$ & 64.3 & 0.31 & 2 & 17810 & 8982.9 & 67.3 & 4.99 \\
\hline 32 & 32768 & 13578.9 & 63.3 & 1.25 & 3 & 40887 & 50393.0 & 65.5 & 2.18 \\
\hline 64 & $262 \mid 44$ & 25908.9 & 63.7 & 0.62 & 4 & 215850 & 454392.0 & 64.5 & 0.62 \\
\hline 128 & $2097 \mid 52$ & 226831.0 & 63.9 & 0.31 & 5 & 313324 & 877980.0 & 64.1 & - \\
\hline
\end{tabular}

FFT: fast Fourier transform; FEM: finite element method.

The elastic and plastic parameters of the solid phase for the two methods are given in Table 1 . The unit cell is subjected to a uniaxial strain tension along its $3 \mathrm{rd}$ axis and the prescribed macroscopic strains are $E_{33}>0, E_{11}=E_{22}=E_{12}=E_{23}=E_{31}=0$.

In order to compare the accuracy and efficiency of these two methods, five different types of mesh for the FEM method and five different sorts of resolution for FFT-based method are performed as shown in the Table 2. All calculations were performed with parallelization of multiple processors on an x64-based Dell computer with eight processors: Intel(R) Core(TM) i7-4790 CPU@3.6 GHz. 

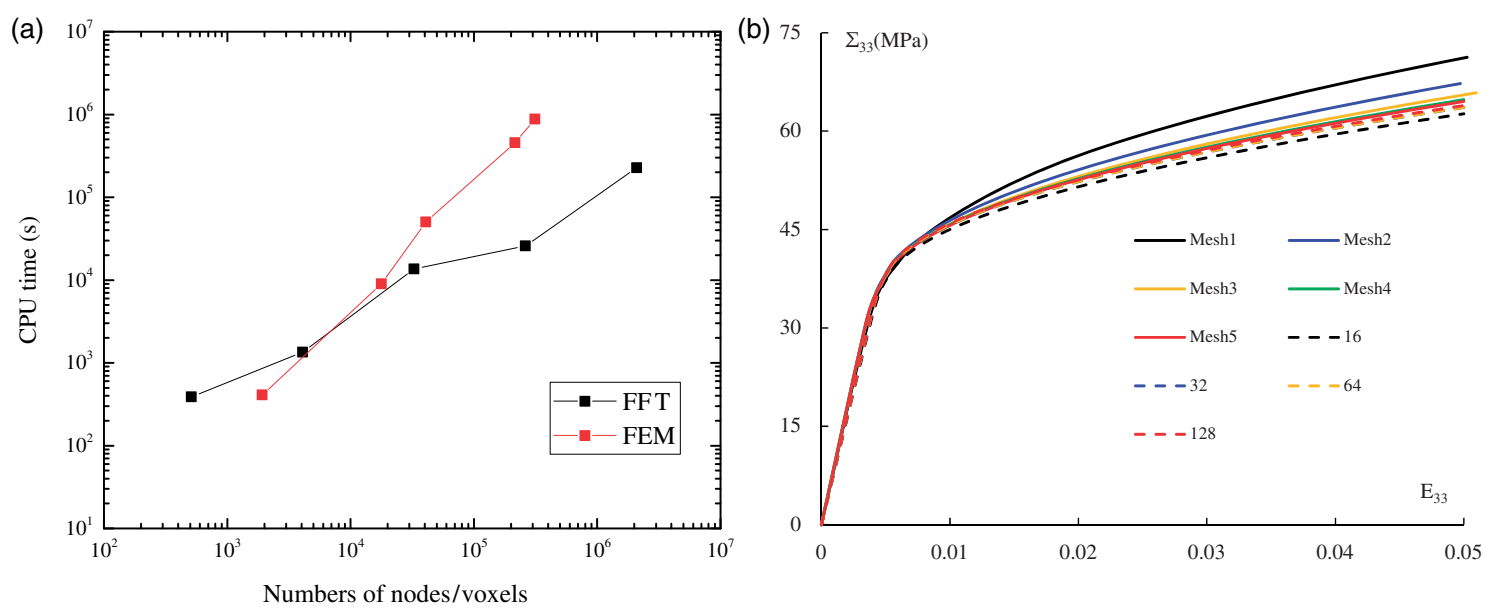

Figure 2. Comparison between FFT method and FEM solution with different meshes and resolutions: (a) CPU time consumption; (b) stress-strain curves.

For the matter of efficiency, the CPU time consumption for different meshes and resolutions is illustrated in Figure 2(a). It is predictably increasing with the nodes and resolution for the two methods, and it is noted that for a low resolution and a small number of nodes, the CPU time consumption of the two methods is approximately at the same level. However, for a higher resolution and a large number of nodes, the FFT methods exhibits a higher efficiency, particularly for the most refined mesh and resolution (last row of Table 2), since the scale of the number of voxels of the FFT method is considerably larger while the CPU time is greatly less. It is also worth noting that the FFT method is able to provide a good accuracy with a relatively low resolution, for example, with a resolution of 32 voxels, which makes the FFT method more efficient since the accuracy of the FEM requires a much larger number of nodes. Therefore, it is obvious that the FFT method is more efficient than the FEM.

The corresponding strain-stress curves are illustrated in Figure 2(b) which clearly indicates that the accuracy of each method strongly depends on the mesh and resolution number, more obviously for the FEM calculations. However, it is noted from this figure that the numerical results for these two methods approach each other as the mesh and resolution are refined, which is specified with the peak stress $\sigma_{p}$ in Table 2. Assuming the FEM calculation with the most refined mesh as the reference solution, one can see that the error of the peak stress for the FEM is decreasing rapidly while it remains at a very small level for all calculations of the FFT methods. In order to illustrate more clearly, the results with the finest mesh and resolution, i.e. the red solid line for the FEM and the red dashed line for the FFT, are taken out from Figure 2(b) and exhibited specifically in Figure 3(a) for a porous medium with a porosity $f=10 \%$. It is noted evidently that the homogenized mechanical response predicted by the FFT-based method is consistent with the one obtained by the FEM, and the accuracy of the FFT-based method is verified (Figure 3(a)). To complete the computational results, the same structure, i.e. a cubic unit cell with a centered spherical void, with a porosity $f=15 \%$ is performed with these two methods. As shown in Figure 3(b), the numerical results of the two methods are in good agreement. 

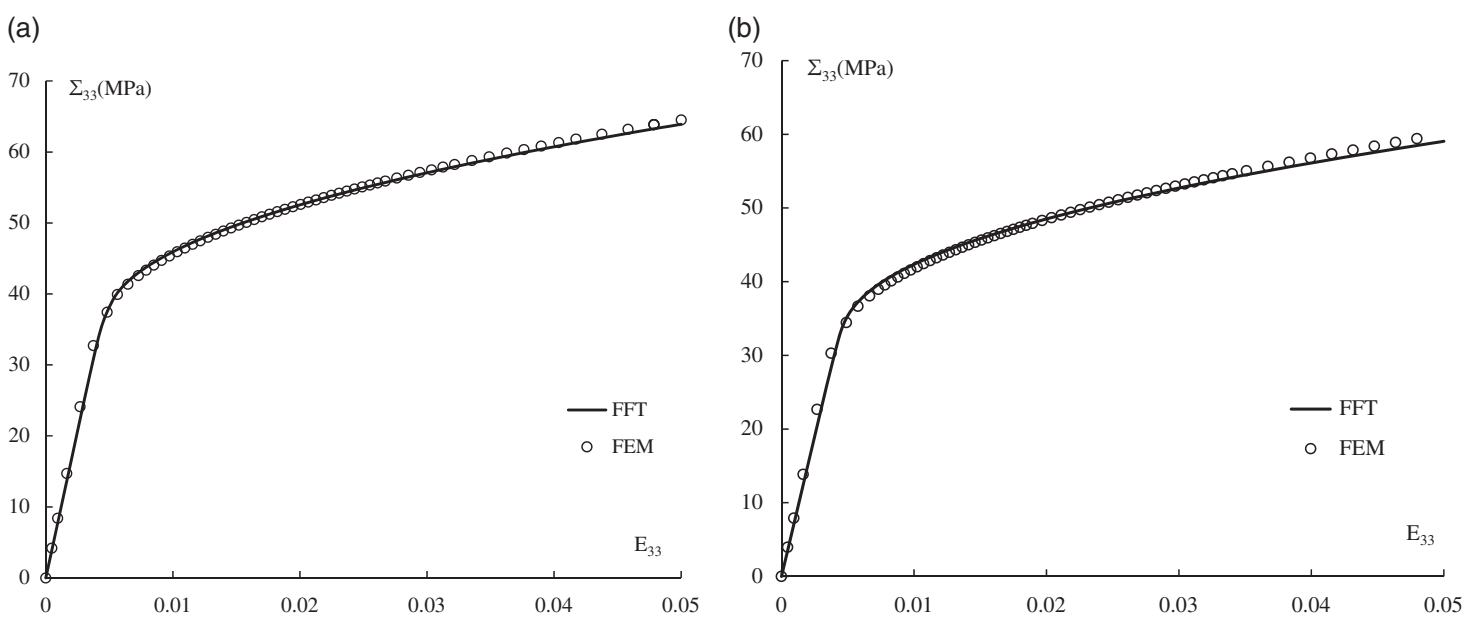

Figure 3. Comparison of the stress-strain curves between the FFT method and the FEM solution with the most fine mesh and resolution: (a) $f=10 \%$; (b) $f=15 \%$.

Table 3. Elastic-plastic parameters of the compressible matrix.

\begin{tabular}{lllllll}
\hline$E(\mathrm{GPa})$ & $v$ & $\sigma_{0}(\mathrm{MPa})$ & $H(\mathrm{MPa})$ & $m$ & $\alpha$ & $\psi$ \\
\hline 28.0 & 0.385 & 8.0 & 180.0 & 0.25 & 1.2 & 0.6 \\
\hline
\end{tabular}

\section{Influence of microstructure on the mechanical behavior of porous material}

Based on the FFT-based numerical model presented in section "FFT-based numerical method", the influences of microstructure on the macroscopic behavior of porous material will be fully studied in this section. The advantage of the FFT-based numerical model is the ability to investigate different kinds of porous geometry (shape, orientation, number) and distribution without fundamental difficulty. For the purpose of further study on porous geomaterials, a pressure sensitive matrix which obeys a Drucker-Prager type criterion with a non-associated plastic flow rule is adopted. The plastic criterion $\phi$ and plastic potential $G$ take the following forms

$$
\begin{aligned}
& \phi=\alpha \sigma_{m}+\sigma_{\mathrm{eq}}-\left(\sigma_{0}+H \gamma^{m}\right) \leq 0 \\
& G=\psi \sigma_{m}+\sigma_{\mathrm{eq}}-\left(\sigma_{0}+H \gamma^{m}\right)
\end{aligned}
$$

in which $\alpha$ and $\psi$ are the frictional and dilatancy coefficients, respectively. $H$ and $m$ are two plastic hardening parameters, and $\gamma$ is the equivalent plastic shear strain in the solid phase.

The volume fractions of the void in the different microstructures are all set to be $f=10 \%$. The same properties of the matrix will be adopted. The corresponding elastic and plastic parameters are given in Table 3. The unit cell is subjected to a uniaxial strain compression along its 3rd axis and the prescribed macroscopic strains $\boldsymbol{E}$

$$
E_{33}<0 \quad E_{11}=E_{22}=E_{12}=E_{13}=E_{23}=0
$$


In order to better show the influence of microstructure on the effective behavior of porous material, we start from the compressible matrix with one pore (spherical or ellipsoidal). After that, a compressible matrix with plenty of pores will be studied.

\section{Porous medium with one centered pore}

Effect of pore shape. In order to consider the effect of pore shape on the macroscopic behavior of the porous medium, three microstructures are generated with different pore shapes in the center. As illustrated in Figure 4, three typical pore shapes are studied: spherical, oblate and prolate. The definition of the aspect ratio of spheroidal pores is shown in Figure $4(\mathrm{~d})$ as $A_{r}=a_{1} / b_{1}$. The ones used in this section are $a_{1} / b_{1}=1 / 2$ for the oblate pore and $a_{1} / b_{1}=2$ for the prolate. The solid phase of the cube obeys a pressure sensitive criterion (16). The unit cell is discretized by a regular grid in three dimensions $128 \times 128 \times 128$.

With a uniaxial strain compression test, the comparisons of the overall responses (stress-strain curve) of porous media with different pore shapes are shown in Figure 5. It can be seen that the effective strength of the porous material with the prolate void is slightly stiffer than the spherical one. The strength reduction with the oblate void is the biggest. In order to better analyze the reason, the vertical stress distributions at the microscopic scale are compared in Figure 6 for these three cases. In Figure 6(a), local stress $\sigma_{33}$ is illustrated for the half of the microstructure with a centered spherical void in three dimensions and the ones in the middle section of the studied cubic cell are shown in Figure 6(b)-6(d) for the three cases. For the spherical void, a stress concentration is clearly observed in the region (blue and purple color) perpendicular to the load direction (equatorial circle). On the

(a)

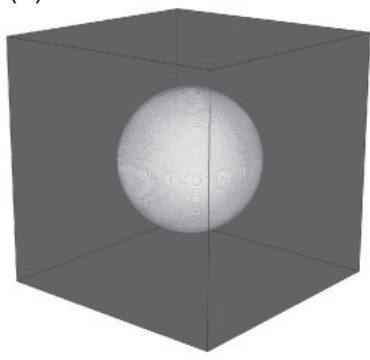

(b)

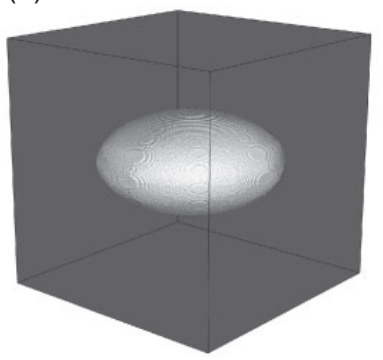

(c)

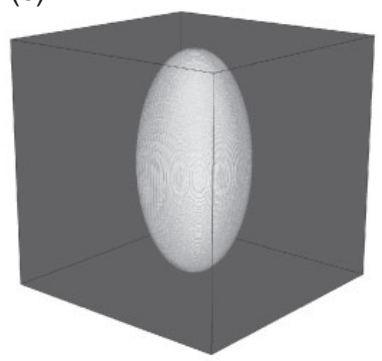

(d)
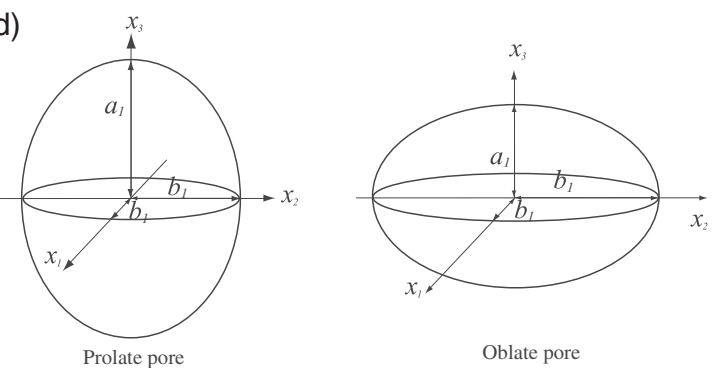

Figure 4. Microstructures used for the FFT simulation with different pore shapes with the same porosity $f=10 \%$ (dark gray: solid matrix; white: pore): (a) spherical: aspect ratio = I; (b) oblate: aspect ratio = I/2; (c) prolate: aspect ratio $=2 ;(d)$ aspect ratio: $A_{r}=a_{1} / b_{1}$. 


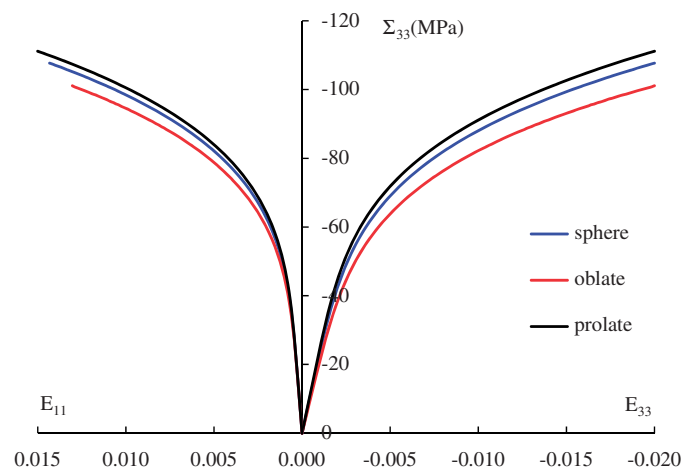

Figure 5. Overall response of porous media with one pore (see Figure 4: spherical, oblate or prolate). Black line: prolate pore; blue line: spherical pore; red line: oblate pore.

(a)

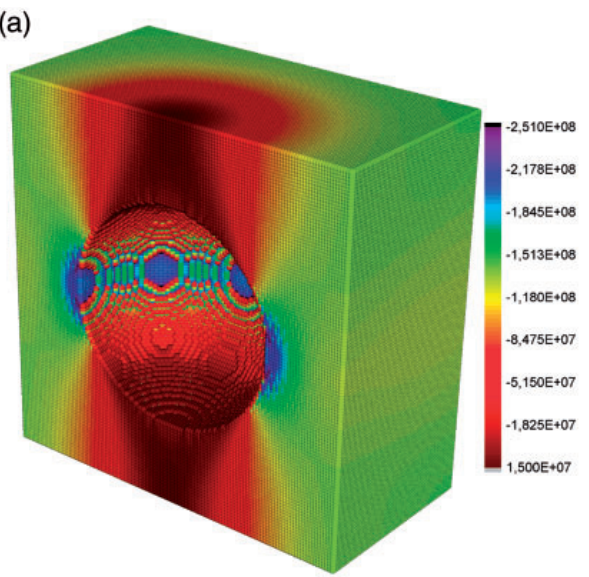

(c)

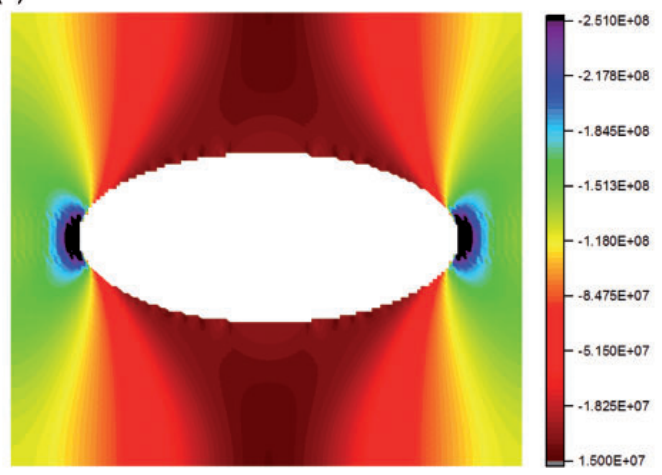

(b)

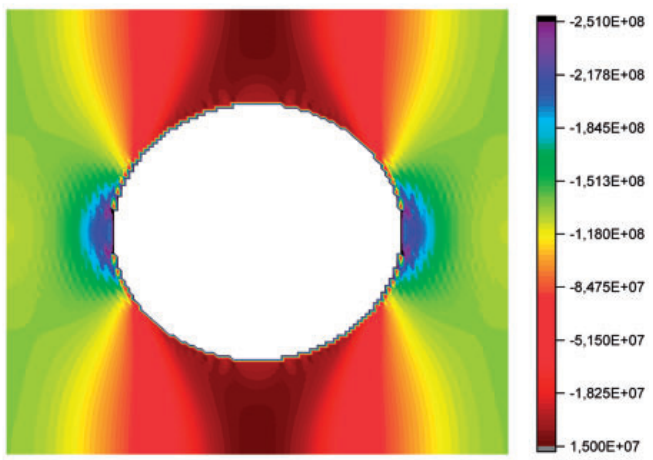

(d)

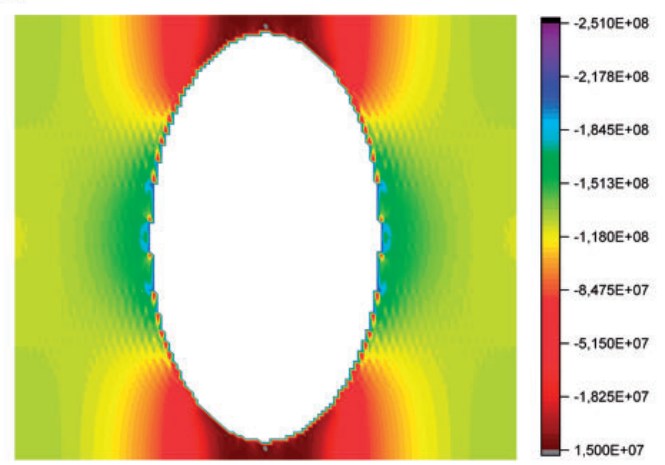

Figure 6. Distributions of microscopic stress $\sigma_{33}(\mathrm{~Pa})$ for different pore shapes: (a) spherical void in three dimensions; (b) spherical void in two dimensions; (c) oblate void in two dimensions; (d) prolate void in two dimensions. 
contrary, the region (red color) along the load direction shows a lower stress level compared with the rest of the matrix (green color). The same phenomenon is noticed for the prolate and oblate voids; however, the difference is the amount of stress concentration and the region with lower stress. It is noted that the region on the equatorial circle of the oblate pore has much more stress concentration but a large amount of the low stress region occurs in the perpendicular direction. Different from the oblate void, the prolate one induces less stress concentration as well as stress dispersion. For the spherical one the stress concentration and dispersion are both moderate. Therefore, considering the stress distribution on the whole field, the overall stress level is lowest for the oblate void but highest for the prolate void.

Effects of aspect ratio of the spheroidal pore. Following the previous analysis, the aspect ratio is definitely another main factor which influences the overall strength of the porous medium. Various aspect ratios of the oblate and prolate pores are therefore considered in the following numerical simulations. The uniaxial strain compression is prescribed in the 33 direction and the numerical results are shown in Figures 7 and 8, respectively.

In general, it can be seen from the two figures that the influence of the aspect ratio for the oblate void is much more obvious than that for the prolate one. For the structure with the oblate pore, the effective strength decreases rapidly with the decrease of the aspect ratio (the smaller the aspect ratio, the flatter the oblate, and one is specific for the spherical void). It is also worth noting that the lateral strain is significantly affected by the aspect ratio, especially for the oblate pore. On the contrary, the aspect ratio has little influence on the overall strength of the structure with the prolate pore. However, slight effects can be also observed.

Unlike the spherical pore, the influences of pore orientation on the macroscopic behavior should be considered for the oblate or prolate pore. For the simplicity, a cubic cell with one oblate or prolate pore with different orientations (the angle between the major axis of the pore and the loading direction: $\theta=0^{\circ}, 45^{\circ}, 90^{\circ}$ ) will be considered. The aspect ratio is the same as the one used in Figure 4

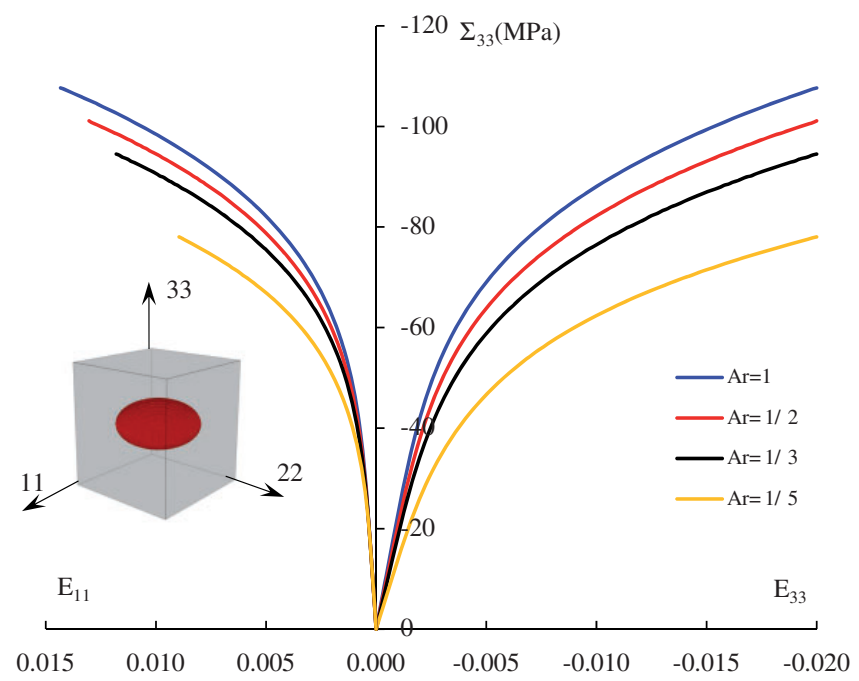

Figure 7. Influence of the aspect ratio of the oblate pore on the overall strength of the porous medium. 


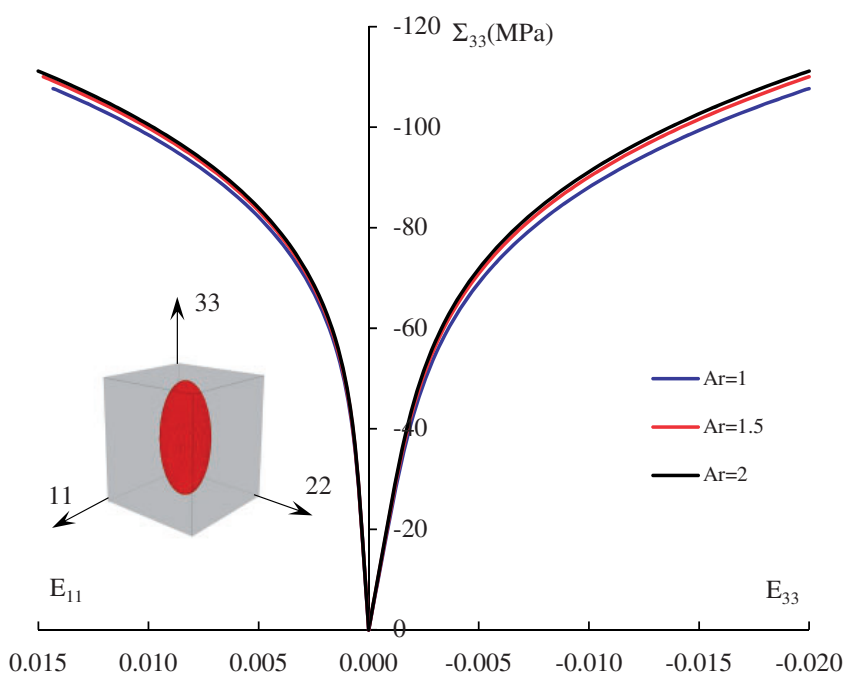

Figure 8. Influence of the aspect ratio of the prolate pore on the overall strength of the porous medium.

for the oblate and prolate pore. The comparisons of the overall responses (stress-strain curve) for the oblate or prolate pore with different orientations are shown in Figure 9(a) and 9(b), respectively. It is noted that the highest strength is obtained for both the oblate and prolate voided materials when the angle $\theta=0^{\circ}$. With $\theta=90^{\circ}$, the lowest strength is found. The influence of oblate void orientation on the effective behavior is larger than for prolate void orientation. The microscopic stress distributions are shown in Figure 10. Stress concentration (blue and purple color) is observed clearly at the boundary of the equatorial plane for all the cases. The stress distributions are different with different microstructures. It is shown that the axial region (red color) possesses lower stress compared with the dominant matrix region (green). For both the oblate and prolate pore, there is a lower stress concentration (blue) and smaller red region in the case of $\theta=0^{\circ}$. The one with $\theta=90^{\circ}$ has a much larger red region compared with other two cases.

\section{Porous medium with randomly oriented and distributed pores}

In porous materials, especially porous geomaterials, the type and distribution of the pores are generally complex. In the representative volume element, different shapes, sizes and orientations may exist simultaneously. Based on the above section for one pore, a cubic cell containing a different number of pores will be studied in this section to consider these effects on the overall behavior by using the FFT-based numerical method. Different kinds of microstructure are generated with different pore shapes and orientations in Figure 11; as an example the porosity is $f=10 \%$ and the number of pores is 40 . For the sake of simplicity, in each microstructure, every pore has the same shape, but the orientation can be different. Figure 11(a) shows a cubic cell with randomly distributed spherical pores with the same radius. The randomly oriented and distributed oblate or prolate pores are illustrated in Figure 11(b) and Figure 11(c), respectively.

The comparisons of the overall strain-stress curve between the three different microstructures are shown in Figure 12 (dashed lines). It can be seen that the strength of the porous medium containing prolate voids is very close to that of the porous medium with spherical voids. The mechanical strength for these two cases is higher than that of the porous medium with oblate pores. This is 

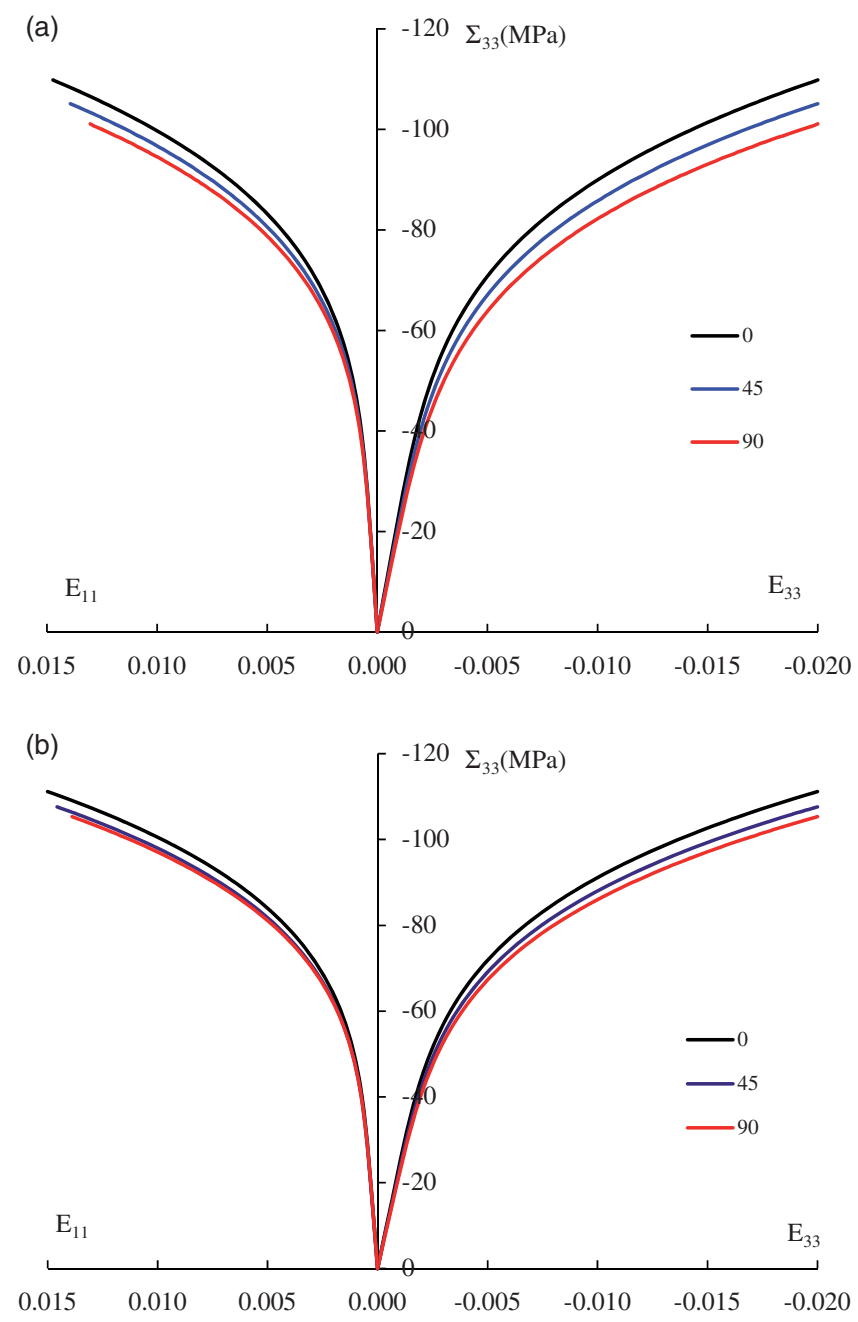

Figure 9. Comparisons of stress-strain curve of a porous material having one oblate or prolate pore with different orientations $\left(\theta=0^{\circ}, 45^{\circ}, 90^{\circ}\right)$ : (a) one oblate void with different orientations; (b) one prolate void with different orientations.

probably due to the fact that the compressive stress is applied perpendicularly to the small axis of oblate pores. These pores behave as open penny-shaped cracks under compressive stress. Compared with the mechanical strength obtained from the cubic cell with one single pore (solid lines) with the same porosity $f=10 \%$, as shown in Figure 12, some differences can be observed. For the cases of prolate and spherical pores, the strength with one single pore is higher than that with 40 pores. However, for the case of oblate pores, the strength with one single pore is slightly lower than that with 40 pores. As the macroscopic strength is obtained from the average volumetric of local stresses, the local stress distributions for the three different distributions of pores are shown in Figure 13. The location of the sections illustrated in Figure 13(b)-13(d) are at the middle plane of the cubic cell (as shown in Figure 13(a)). The basic difference between the case with one single pore and that with 40 pores is the interaction effect between pores. However, in view of the results presented here, it is not 
(a)

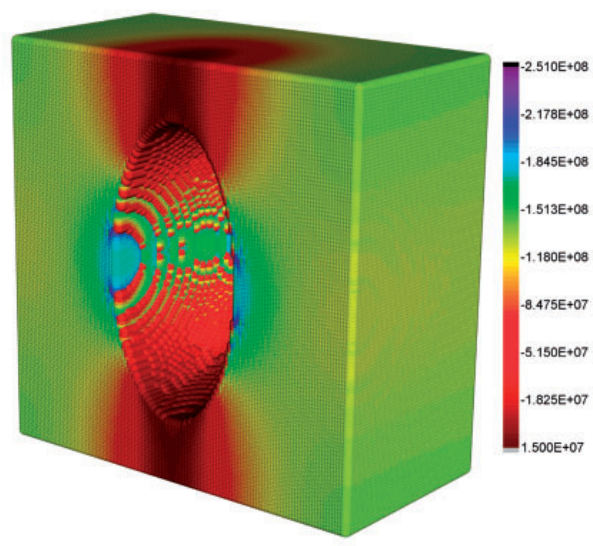

(c)

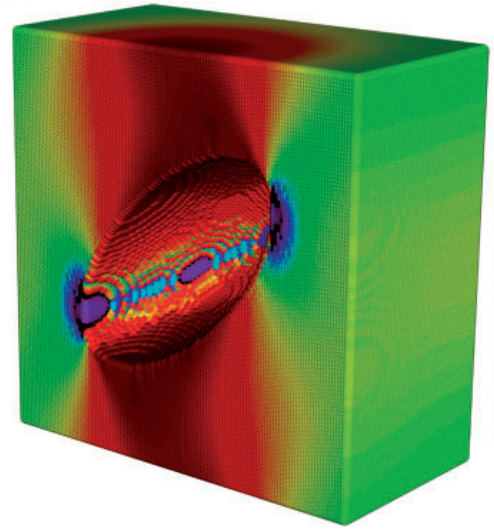

(e)

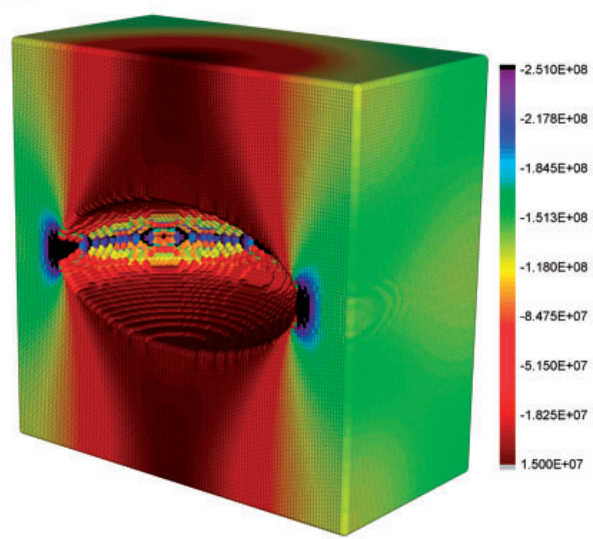

(b)

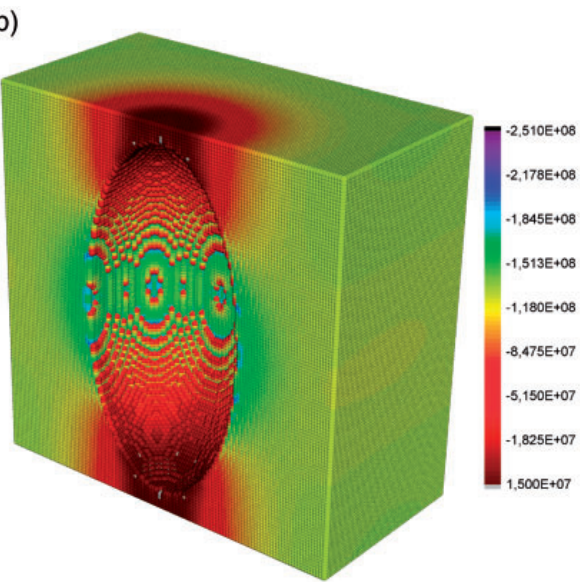

(d)

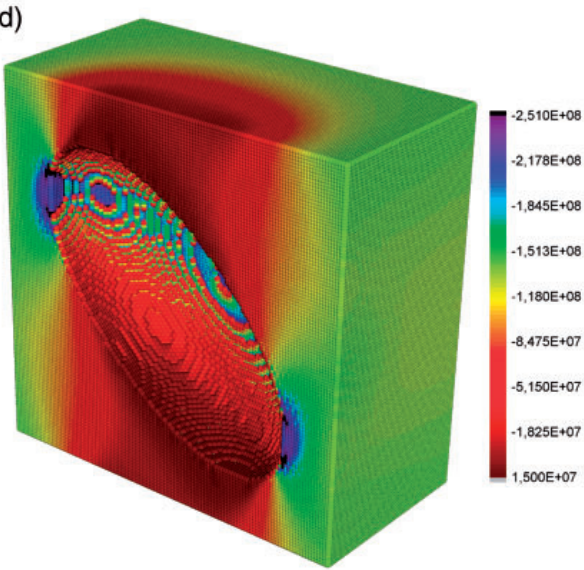

(f)

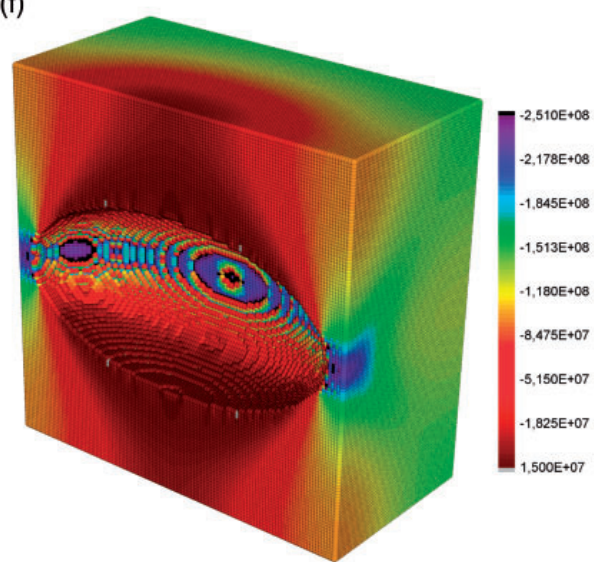

Figure 10. Distribution of microstress $\sigma_{33}(\mathrm{~Pa})$ in the cubic cell having a oblate or prolate pore with different orientations $\theta$ : (a) oblate $\theta=0^{\circ}$; (b) prolate $\theta=0^{\circ}$; (c) oblate $\theta=45^{\circ}$; (d) prolate $\theta=45^{\circ}$; (e) oblate $\theta=90^{\circ}$; (f) prolate $\theta=90^{\circ}$. 


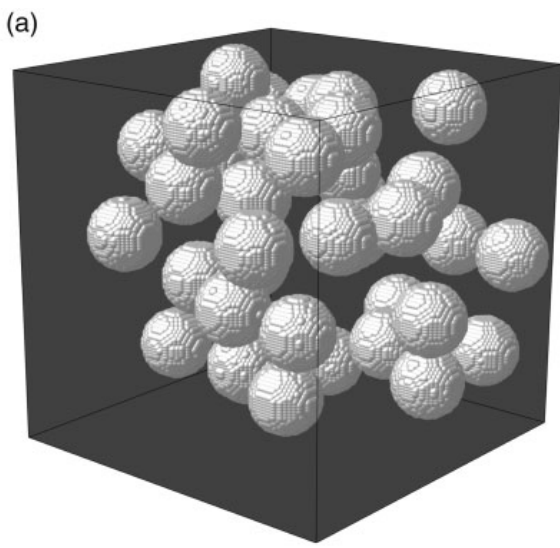

(b)

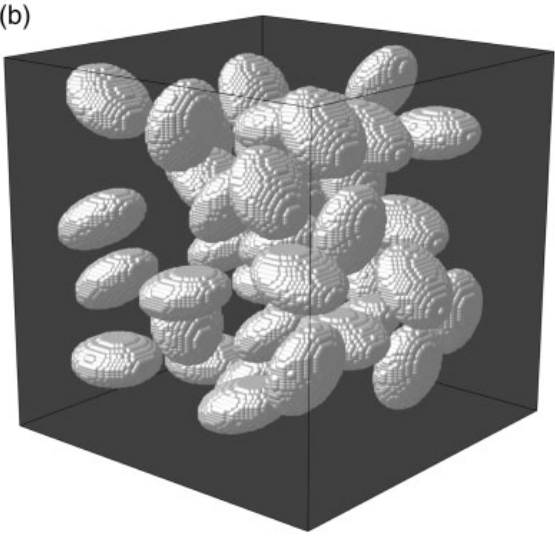

(c)

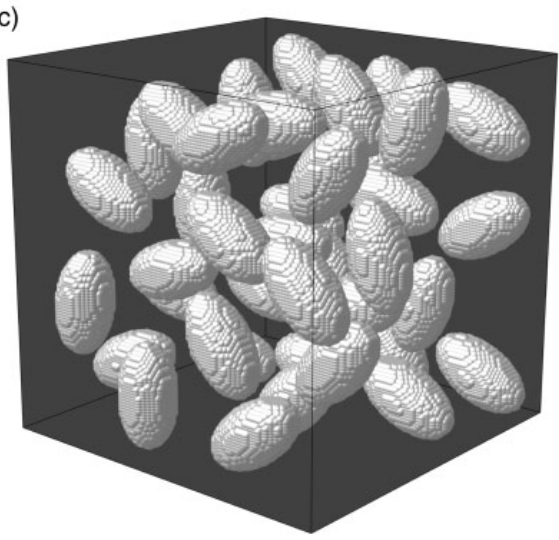

Figure I I. Microstructures with randomly oriented distributed pores: (a) spherical void; (b) oblate void; (c) prolate void. Oblate pore: aspect ratio $=1 / 2$; Prolate pore: aspect ratio $=2$.

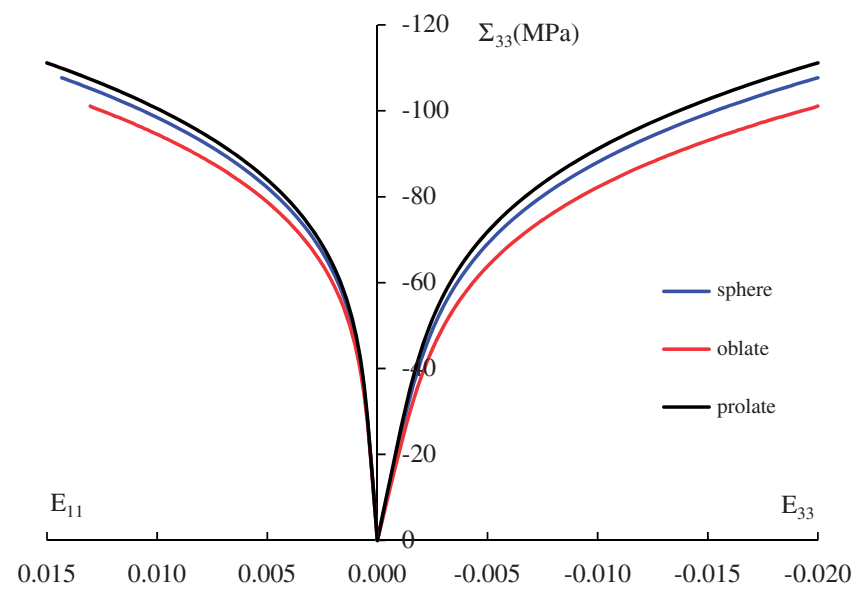

Figure 12. Comparisons of stress-strain curve for porous materials with randomly distributed voids. Solid line: microstructure in Figure 4; dashed line: microstructure in Figure II. 
(a)

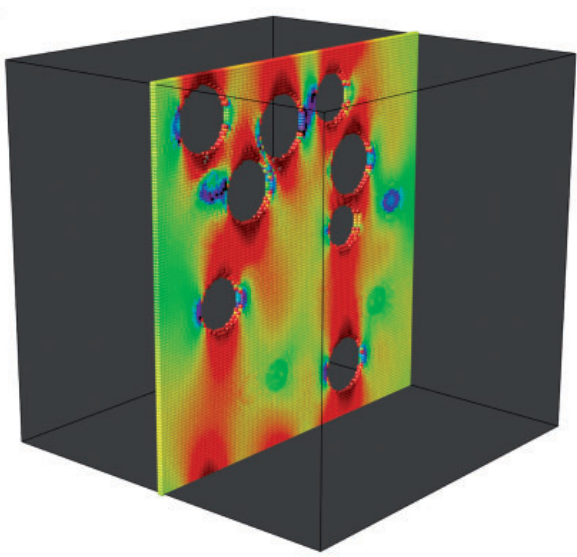

(c)

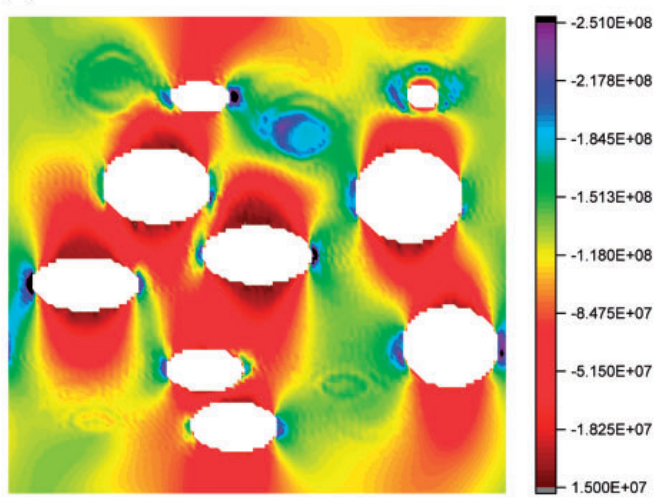

(b)

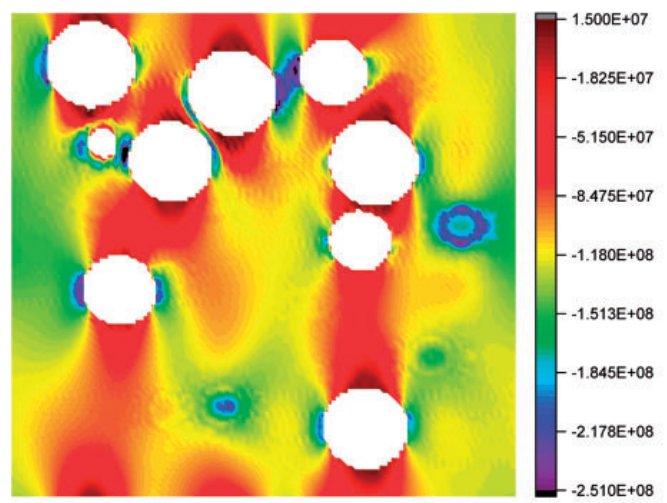

(d)

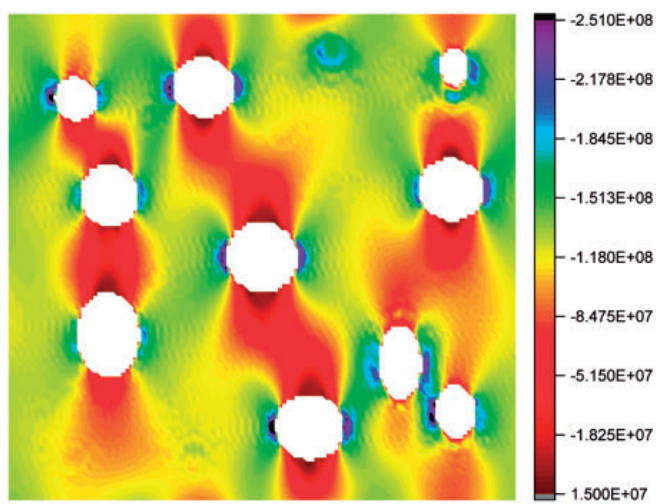

Figure 13. Distributions of microscopic stress $\sigma_{33}(\mathrm{~Pa})$ for different randomly distributed voids: (a) spherical voids in three dimensions; (b) spherical voids in two dimensions; (c) oblate voids; (d) prolate voids.

easy to give a definite conclusion about this interaction effect. It seems that the interaction effect depends on the pore shape and loading path. For the particular case of uniaxial strain compression studied here, it seems that the interaction between pores induces a compensation effect of local stresses for the porous materials with spherical and prolate pores while a very small amplification effect is observed for that with oblate pores. As mentioned in Kachanov (1994), the impact of interactions on the effective moduli decreases as porosity decreases. The distribution and aspect ratio of elliptical pores have also influences on the interaction. Further studies are necessary to capture a general trend of interaction effects by performing an statistical analysis by considering a high number of microstructures and different loading paths.

\section{Extension to a damage model}

In the above sections, a FFT-based numerical model is proposed to study the effects of pores on the macroscopic behavior of porous materials. With the increase of loading, the plastic deformation will accumulate. According to some microscopic analyses with digital image correlation techniques, microcracks can initiate and propagate in the porous material when the stress or plastic deformation 
exceeds a critical value. The microcracking process affects the mechanical behavior. In order to take into account the damage generated during the loading, a simple damage model will be proposed in this section based on the FFT technique.

The main advantage of the FFT-based numerical approach is that it is able to efficiently consider non-regular geometrical forms of microstructure. The heterogeneous material field is discretized into a series of grid points. Each point has a mechanical property according to its location in the heterogeneous microstructure. Taking advantage of these properties of the FFT-based approach, we assume that the point located in the solid matrix will lose its capability of supporting force when the accumulated plastic deformation $\gamma$ in this point exceeds a critical value $\epsilon_{0}$

$$
\gamma \geq \epsilon_{0}
$$

That is to say, the properties in these points satisfying the condition (18) will be replaced by the ones of pore. In the present study, the criterion (18) is adopted for the purpose of simplicity. The growth and coalescence of pores are all controlled by this law during the compression tests. However, based on this trial work, other more reasonable, efficient and realistic damage evolution laws can be proposed in the future work.

\section{Damage model with one centered pore}

In order to evaluate the proposed damage model, it is first applied to the porous material with one centered pore. Following the previous analysis, the microstructures with oblate, spherical and prolate pores are thoroughly investigated and the numerical results are shown in Figure 14. For the simplicity of comparison and analysis, all results are performed with the same damage threshold $\epsilon_{0}=0.015$. In order to clearly show the effects of the damage process, the undamaged strain-stress curves (red lines) are presented simultaneously with the damaged ones (black lines). At the same time, the damage evolution is illustrated at different stages of the loading process at points A, B, C, D. It can be observed from the figures that the strain-stress curve of the damage material remains exactly the same as the undamaged one before the damage occurs. As soon as damage takes place, the curve starts deviating from the red line (sound materials) but still increases until the point $\mathrm{B}$. It is implied that the structure does not lose its capacity at once but failure occurs when the damage zone largely develops around the whole pores (see Figure 14 of damage evolution at point B). Furthermore, it is indicated that the medium with oblate pores commences damage earlier and the one with prolate pores occurs aftermost which is consistent with the previous analysis that stress concentration is more severe for the medium with oblate pores, and the damage propagates exactly at that region. Moreover, the damage grows perpendicular to the loading direction which is also in good agreement with the former analysis. Beside of the suddenly fall (starts at point B for all cases) due to the simple damage criterion, the strain-stress curve shows that the medium with oblate pore is more ductile as can be seen from Figure 14(a) that the damage propagates gradually before point B.

\section{Damage model with randomly distributed pores}

With the same procedure the materials with randomly distributed voids are investigated in this section. The strain-stress curves are presented for both sound materials and damaged materials with damage evolution at different stages of the loading process at points A, B, C, D (see Figure 15). On the contrary, in the case of a porous medium with one pore, it is indicated that the damage strain-stress curves for the microstructure with randomly distributed pores are quite similar, for 

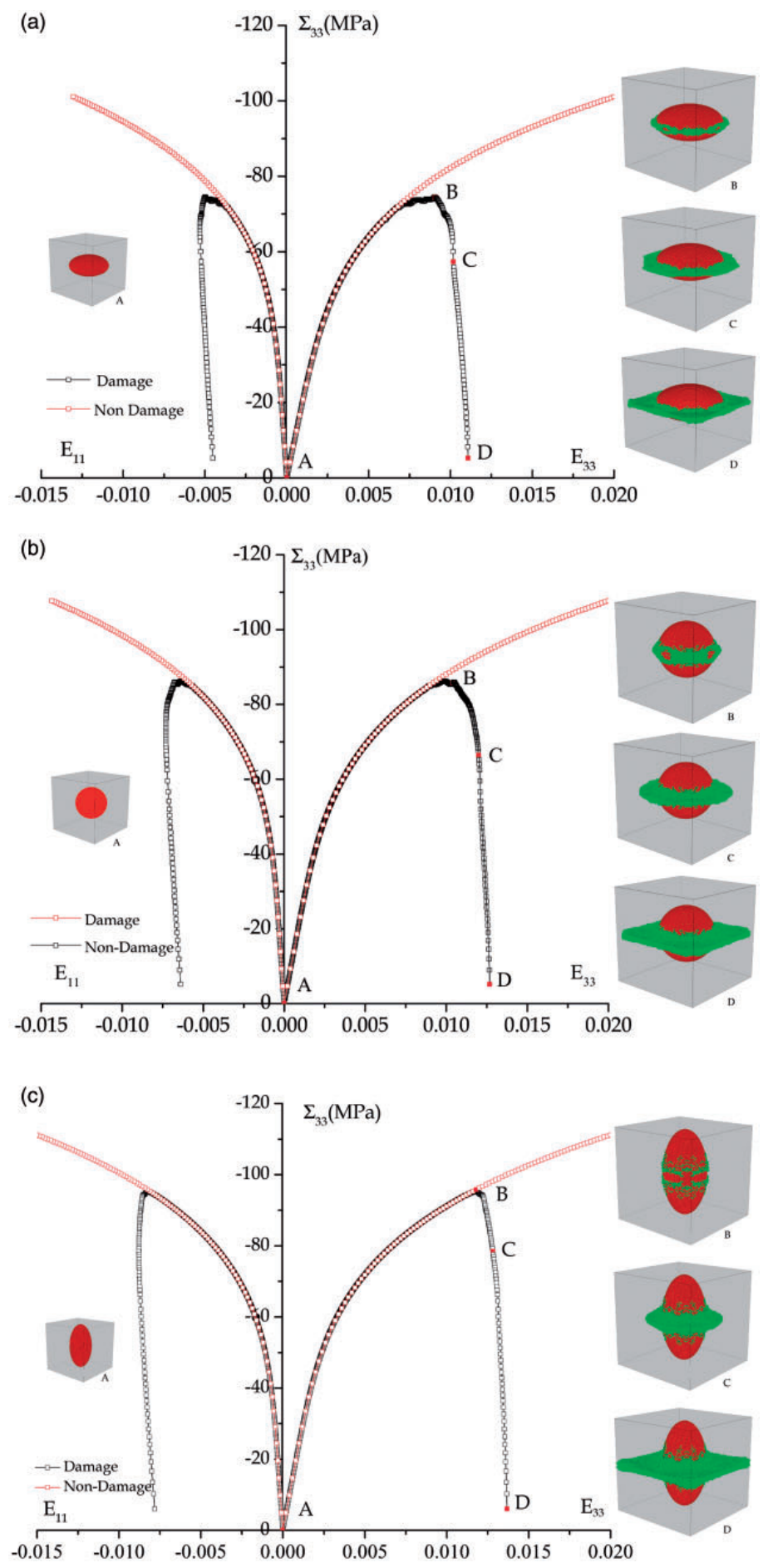

Figure 14. The damage evolution in the porous medium with one centered pore: (a) oblate pore; (b) spherical pore; (c) prolate pore. 

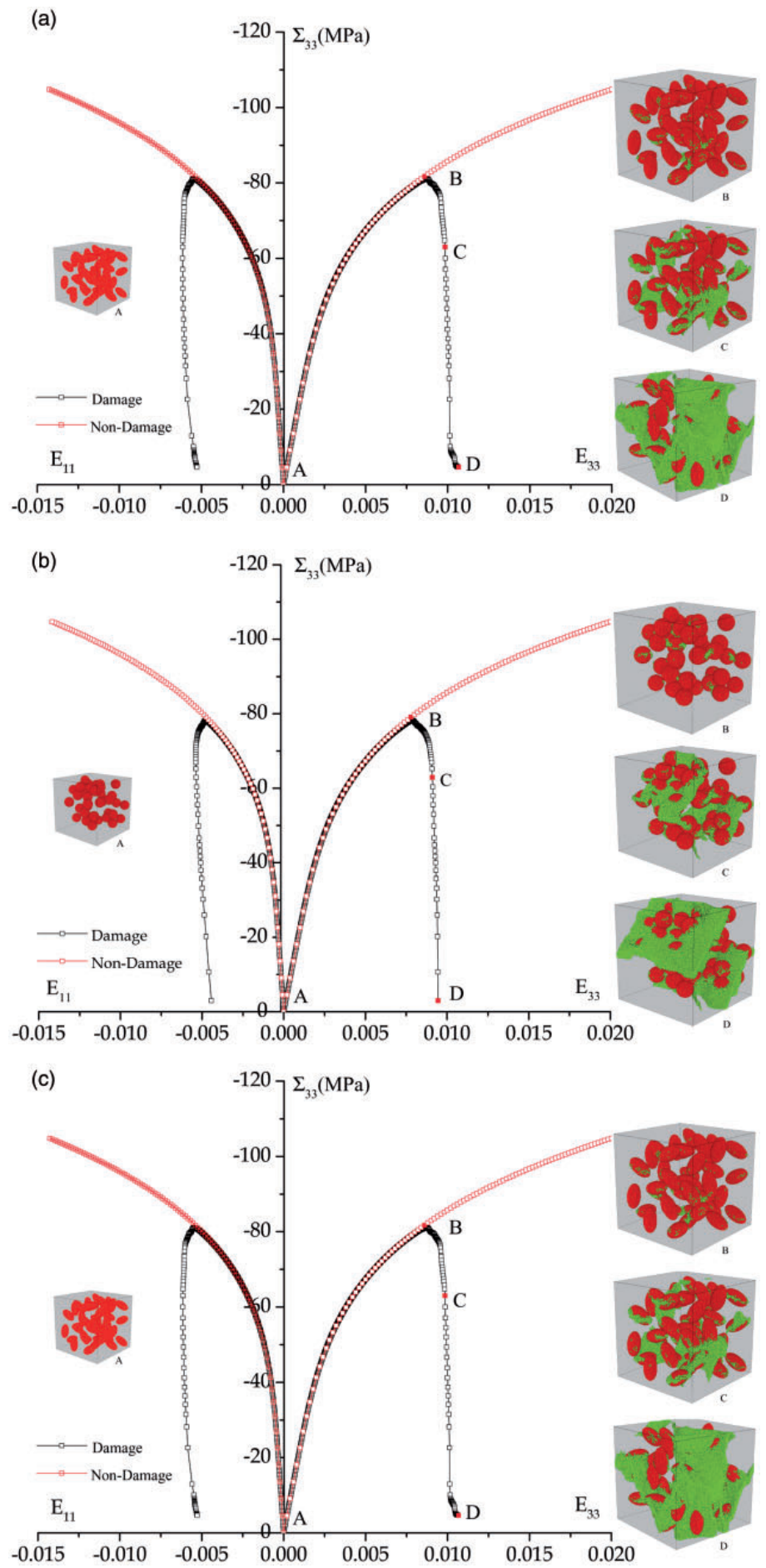

Figure 15. Porous medium with randomly distributed pores with damage evolution: (a) oblate pores; (b) spherical pores; (c) prolate pores. 
example, the point of the damage occurrence, the process of damage growth and the final stage. From the viewpoint of microstructure evolution, the three cubic figures in the right show that very little damage first takes place among different pores (point B). After a few increments of loading the damage largely develops and coalesces (point C). Afterwards, the damage grows rapidly until the region without distribution of pores (point D). In other words, the random distribution of pores reduces the influences of the void shape. Furthermore, compared with the same shape with one centered pore (see Figure 14), the results of randomly distributed pores show less peak strength respectively which indicates that the randomly distributed voids accelerate the damage process since the stress interactions are more complex than the analysis in the previous sections.

\section{Application to the sandstone with a simple damage criterion}

The proposed damage model will be adopted to describe the mechanical behavior of "Vosges sandstone" which comes from the Vosges mountains in France. The sandstone is a typical porous quasibrittle rock which is mainly composed of quartz grains $(93 \%)$, with a porosity $f=20 \%$. With the increase of confining pressure, its mechanical behavior exhibits a brittle-ductile transition from low to high confining pressures (Besuelle et al., 2000; Menendez et al., 1996). The distribution of pores in the sandstone is generally complex. In an ideal approach, the Representative Volume Element (RVE) used for numerical modeling should be chosen from three-dimensional segmented images issued from a microscopic analysis, for instance using a microtomography technique. However, such data are not available for the studied sandstone. Furthermore, at this stage of study, the emphasis is put on the verification of the FFT method efficiency for porous rock-like materials with plastic deformation and damage processes. Therefore, the RVE is simply generated in a numerical way with randomly distributed and uniformly sized pores to characterize the basic information of the real sandstone. The cubic unit cell is discretized by a regular grid of points $(128 \times 128 \times 128)$ and contains 40 pores, as shown in Figure 16.

As in the previous work by Jiang and Shao (2012), a non-associated Drucker-Prager model is adopted to characterize the mechanical behavior of the solid matrix. The plastic criterion $\phi$ and potential $G$ with an isotropic plastic hardening function $R(\gamma)$ are given in the same form as equation

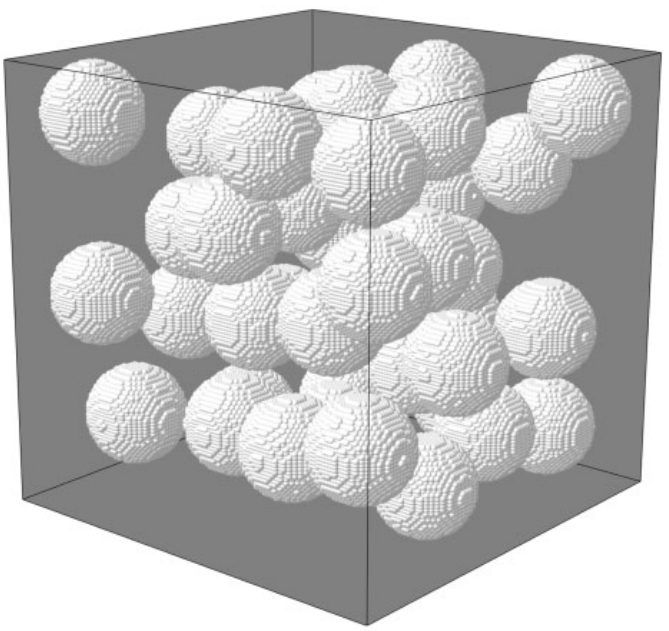

Figure 16. The representative elementary volume of porous sandstone with a porosity $f=20 \%$. 
(16). In order to perform numerical modeling of the macroscopic behavior of sandstone, the elastic and plastic parameters of the solid phase should be first determined. The direct identification of the constituent properties in heterogeneous materials from relevant experimental data is still an open challenge which is clearly not the ambition of the present paper. No experimental data are available in terms of local stresses and strains for the sandstone. We have adopted an iterative numerical optimization technique. Knowing the porosity, the local elastic and plastic parameters of the solid phase are iteratively fitted from the macroscopic stress-strain curves obtained from a triaxial compression test with $5 \mathrm{MPa}$ confining pressure and the obtained values are given in Table 4 . However, it

Table 4. Elastic-plastic parameters of the sandstone used in the simulations.

\begin{tabular}{lllllll}
\hline$E(\mathrm{GPa})$ & $v$ & $\sigma_{0}(\mathrm{MPa})$ & $H(\mathrm{MPa})$ & $m$ & $\alpha$ & $\psi$ \\
\hline 28.0 & 0.385 & 17.0 & 180.0 & 0.25 & 1.2 & 0.6
\end{tabular}
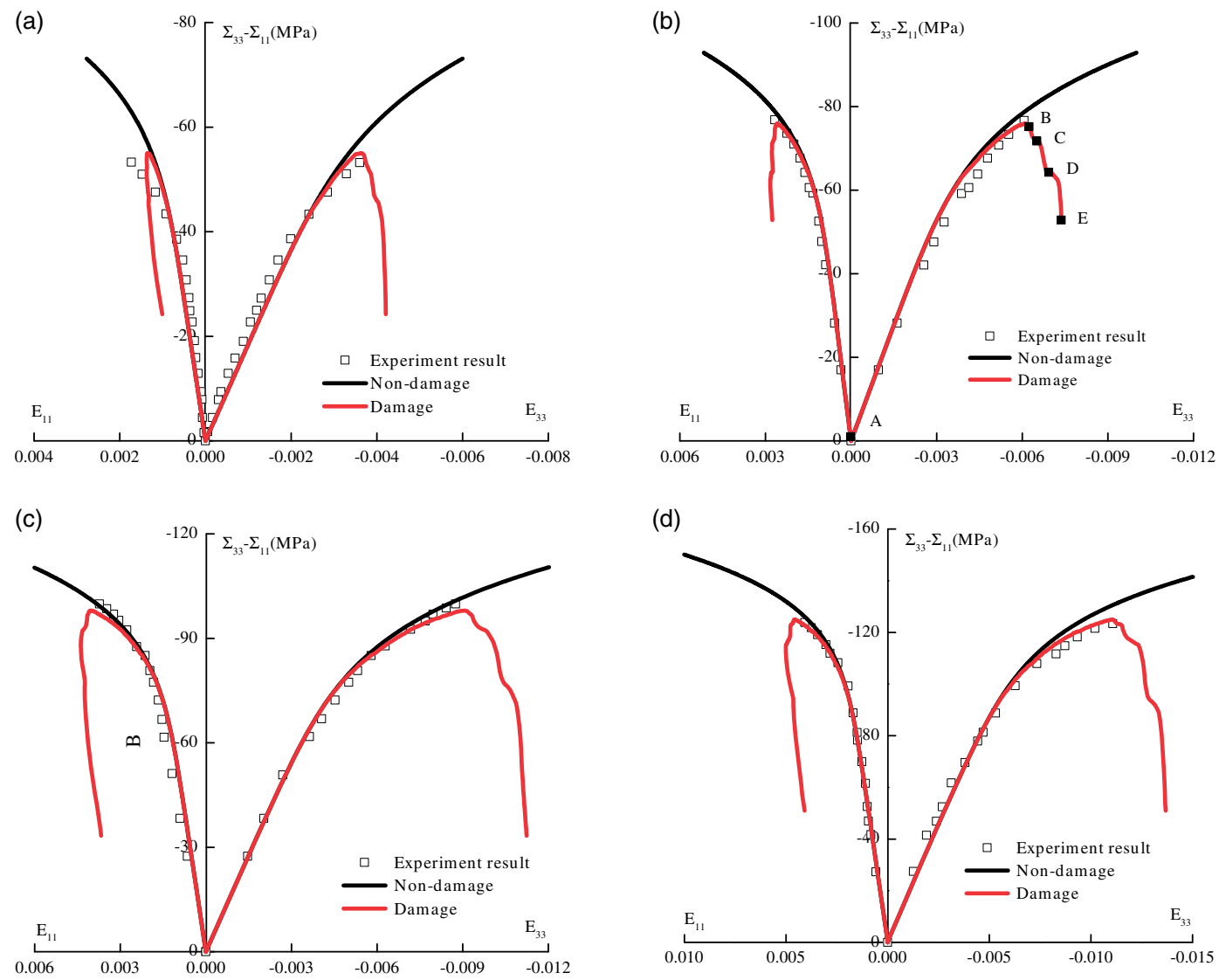

Figure 17. Comparisons between the results predicted by FFT-based damage model and experimental data of sandstone with different confining pressures: (a) $5 \mathrm{MPa} \epsilon_{0}=0.0065$; (b) $10 \mathrm{MPa} \epsilon_{0}=0.0155$; (c) $20 \mathrm{MPa} \epsilon_{0}=0.0275$; (d) $40 \mathrm{MPa} \epsilon_{0}=0.0420$. 
is important to point out that the same values of parameters are used for all other tests with different confining pressures.

Using the same set of elastic and plastic parameters, the numerical predictions for undamaged and damaged materials are compared with experimental data as shown in Figure 17 with different confining pressures $(5,10,20$ and $40 \mathrm{MPa})$. However, it is well known that the confining pressure has a great influence on the mechanical behavior of rock-like materials such as sandstone. With the increase of confining pressure, the initial damage threshold generally increases such as for the initial plastic yield stress and the peak strength which are determined by a pressure-dependent plastic criterion. It is also needed to determine an appropriate damage initiation criterion taking into account such a confining pressure effect. For the sake of simplicity in the present work, different values of the damage threshold $\epsilon_{0}$ are simply used for different confining pressures. This aspect should be improved in our future work by the identification of a pressure-dependent damage

(a)

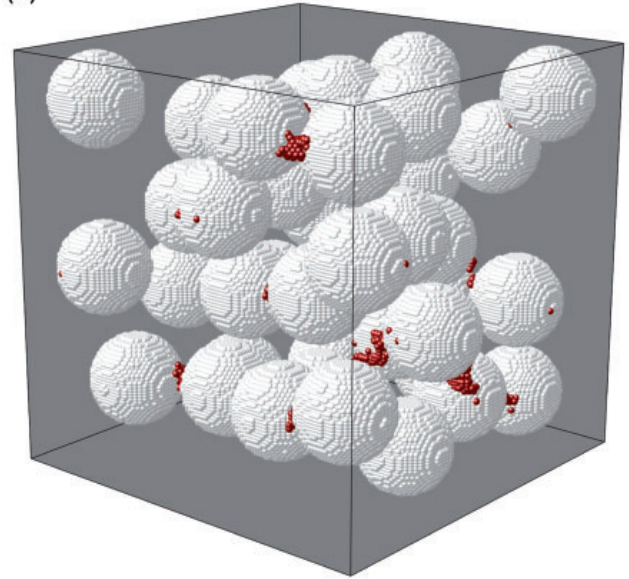

(c)

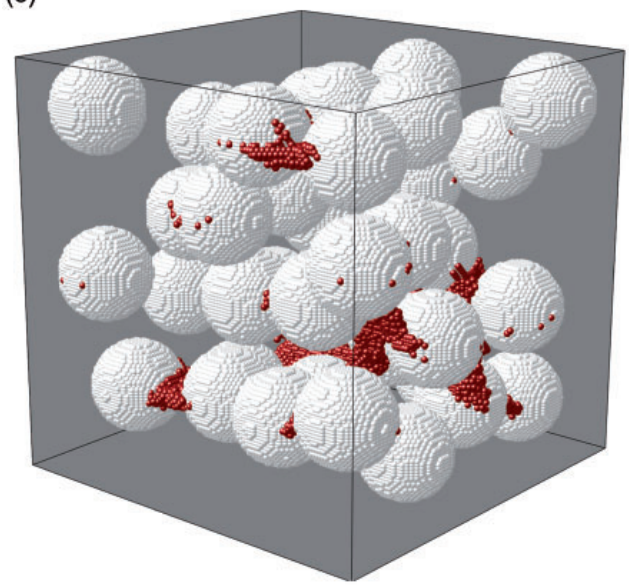

(b)

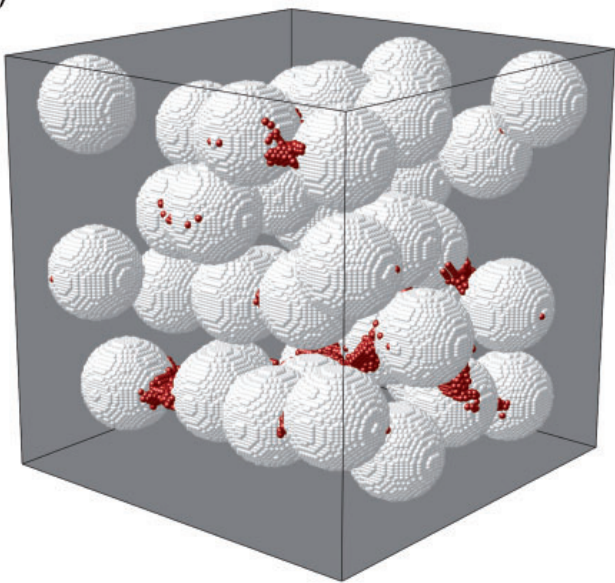

(d)

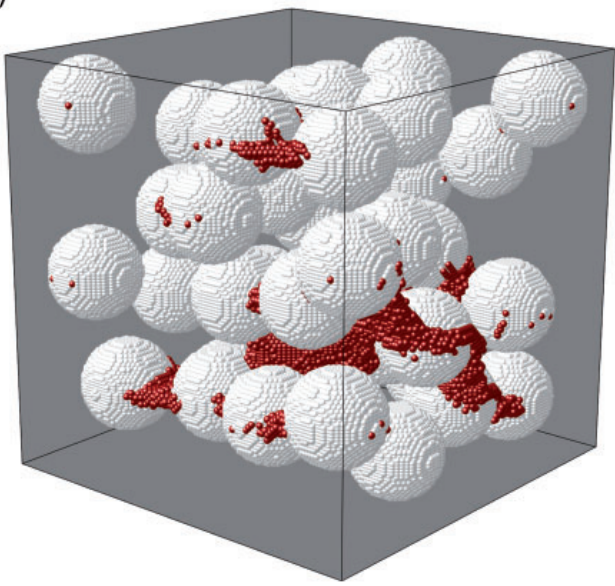

Figure 18. Evolution of damage generated in the microstructure: (a) Stage I (Point B); (b) Stage 2 (Point C); (c) Stage 3 (Point D); (d) Stage 4 (Point E). 
criterion. With this method, both the lateral and axial strains are well predicted by the proposed numerical model for all confining pressures.

It can be seen from Figure 17 that the numerical predictions of undamaged models (black lines) are in good agreement with the experimental data (points) in the forepart of the curve but generally provide a much too stiff response when the stress becomes higher. On the contrary, the numerical calculations of damaged models (red lines) are generally consistent with the entire experimental data thanks to the induced damage process. To be more specific, one takes a compression test with $10 \mathrm{MPa}$ confining pressure (Figure 17(b)) as an example. One can clearly observe that the red line (with damage) is lower and closer to the experimental data than the black line (without damage) even before the peak stress point $\mathrm{B}$. That means that the damage process occurs simultaneously with the plastic process before the peak point $\mathrm{B}$ and the mechanical behavior of the porous material before and after the peak stress state. The evolutions of damage generated in the microstructure at different stages corresponding to the points B, C, D and E at the stress-strain curve are illustrated in Figure 18. Further, with the FFT-based numerical method, the damage propagation and coalescence are clearly observed. At a primary stage (Figure 18(a)), a small damage zone (red part) is generated at the nearby region of pores and afterwards the coalescence of damage zone occurs at the space of pore clustering (Figure 18(b)-18(c)). At the final stage (Figure 18(d)), an extensive coalescence zone is formed between different clusters of pores. These connected damaged regions make the whole field degraded. However, with only one chosen microstructure studied here, one should be careful in providing a general conclusion about the damage evolution kinetics inside the porous material.

\section{Conclusion}

A FFT-based micromechanical model has been proposed in this work for the description of plastic deformation and damage processes in porous rock-like material. The accuracy and efficiency of the FFT-based micromechanical model for porous materials is first checked by comparing with FEM numerical results. Without the need for mesh generation, the FFT-based model has the advantage of capturing complex distributions of pores or other heterogeneities. Different microstructures have been considered for investigating the effects of pore shape, number, size and orientation on the macroscopic behavior of the studied porous material. It is found that for the porous material with one single pore under uniaxial compression, the mechanical strength for spherical and prolate pores is higher than that for oblate pores. When a random distribution of pores is considered, the effect of interaction between pores also depends on the pore shape. For spherical and prolate pores, the interaction induces a compensation effect in terms of peak strength while an amplification effect is observed for oblate pores. The emphasis of the present study was then put on the induced damage process in rock-like porous materials. Even a very simple damage criterion has been used to capture the creation of new pores, it was possible to get some interesting results on the damage evolution kinetics. It is found that damage generally initiates from regions close to pore surfaces and then propagates inside the solid matrix. The macroscopic failure of porous materials is inherently related to the coalescence of pores around the peak stress region. In order to check the ability of the micromechanical model to reproduce experimental data, the mechanical behavior of a typical sandstone under triaxial compression tests has been investigated using the proposed model. In a general way, there was a good agreement between the numerical results and experimental data for both axial and lateral strains with different confining pressures. However, a number of improvements should be performed in future works. A statistical analysis needs to be performed considering a large number of microstructures in order to obtain a better understanding of the interaction effects of pore 
distribution. A pressure-dependent damage criterion should also be proposed. As a further ambition, segmented three-dimensional images directly issued from microscopic analyses should be used in FFT-based numerical simulations in order to account for the real microstructures of the materials.

\section{Declaration of Conflicting Interests}

The author(s) declared no potential conflicts of interest with respect to the research, authorship, and/or publication of this article.

\section{Funding}

The author(s) received no financial support for the research, authorship, and/or publication of this article.

\section{References}

Anoukou K, Pastor F, Dufrenoy P, et al. (2016) Limit analysis and homogenization of porous materials with Mohr-Coulomb matrix. Part I: Theoretical formulation. Journal of the Mechanics and Physics of Solids 91: 145-171.

Benzerga A, Besson J and Pineau A (1999) Coalescence-controlled anisotropic ductile fracture. Journal of Engineering Materials and Technology 121(2): 221-229.

Besuelle P, Desrues J and Raynaud S (2000) Experimental characterisation of the localisation phenomenon inside a vosges sandstone in a triaxial cell. International Journal of Rock Mechanics and Mining Sciences 37(8): 1223-1237.

Bilger N, Auslender F, Bornert M, et al. (2005) Effect of a nonuniform distribution of voids on the plastic response of voided materials: a computational and statistical analysis. International Journal of Solids and Structures 42(2): 517-538.

Cazacu O and Stewart JB (2009) Analytic plastic potential for porous aggregates with matrix exhibiting tension-compression asymmetry. Journal of the Mechanics and Physics of Solids 57(2): 325-341.

Cheng L, De Saxce G and Kondo D (2014) A stress-based variational model for ductile porous materials. International Journal of Plasticity 55: 133-151.

Durban D, Cohen T and Hollander Y (2010) Plastic response ofporous solids with pressure sensitive matrix. Mechanics Research Communications 37(7): 636-641.

Eyre DJ and Milton GW (1999) A fast numerical scheme for computing the response of composites using grid refinement. The European Physical Journal Applied Physics 6(1): 41-47.

Fritzen F, Forest S, Böhlke T, et al. (2012) Computational homogenization of elasto-plastic porous metals. International Journal of Plasticity 29: 102-119.

Garajeu M and Suquet P (1997) Effective properties of porous ideally plastic or viscoplastic materials containing rigid particles. Journal of the Mechanics and Physics of Solids 45(6): 873-902.

Gologanu M, Leblond J and Devaux J (1993) Approximate models for ductiles metals containing non-spherical voids - case of axisymmetric prolate ellipsoidal cavities. Journal of the Mechanics and Physics of Solids 41(11): 1723-1754.

Gologanu M, Leblond J and Devaux J (1994) Approximate models for ductile metals containing non-spherical voids-cas of axisymmetric oblate ellipsoidal cavities. ASME Journal of Engineering Materials and Technology 116(3): 290-297.

Gologanu M, Leblond J, Perrin G, et al. (1997) Recent extensions of gurson's model for porous ductile metals. Continuum Micromechanics 377: 61-130.

Guo T, Faleskog J and Shih C (2008) Continuum modeling of a porous solid with pressure-sensitive dilatant matrix. Journal of the Mechanics and Physics of Solids 56(6): 2188-2212.

Gurson A (1977) Continuum theory of ductile rupture by void nucleation and growth: part1-yield criteria and flow rules for porous ductile media. ASME Journal of Engineering Materials and Technology 99(1): 2-15. 
Jeong H (2002) A new yield function and a hydrostatic stress-controlled model for porous solids with pressuresensitive matrices. International Journal of Solids and Structures 39(5): 1385-1403.

Jiang T and Shao JF (2012) Micromechanical analysis of the nonlinear behavior of porous geomaterials based on the fast fourier transform. Computers and Geotechnics 46: 69-74.

Kachanov M (1994) Elastic solids with many cracks and related problems. Advances in Applied Mechanics 30: $259-445$.

Keralavarma S and Benzerga A (2010) A constitutive model for plastically anisotropic solids with non-spherical voids. Journal of the Mechanics and Physics of Solids 58(6): 874-901.

Leblond J, Perrin G and Suquet P (1994) Exact results and approximate models for porous viscoplastic solids. International Journal of Plasticity 10(3): 213-235.

Lee $\mathbf{J}$ and Oung $\mathbf{J}$ (2000) Yield functions and flow rules for porous pressure-dependent strain-hardening polymeric materials. Journal of Applied Mechanics 67(2): 288-297.

Maghous S, Dormieux L and Barthèlèmy J (2009) Micromechanical approach to the strength properties of frictional geomaterials. European Journal of Mechanics A/Solid 28(1): 179-188.

Menendez B, Zhu W and Wong TF (1996) Micromechanics of brittle faulting and cataclastic flow in berea sandstone. Journal of Structural Geology 18(1): 1-16.

Michel JC and Suquet P (1992) The constitutive law of nonlinear viscous and porous materials. Journal of the Mechanics and Physics of Solids 40(4): 783-812.

Michel JC, Moulinec H and Suquet P (1999) Effective properties of composite materials with periodic microstructure: a computational approach. Computer Methods in Applied Mechanics and Engineering 172(1): $109-143$.

Michel JC, Moulinec H and Suquet P (2000) A computational method based on augmented lagrangians and fast fourier transforms for composites with high contrast. Computer Modeling in Engineering and Sciences 1(2): 79-88.

Michel JC, Moulinec H and Suquet P (2001) A computational scheme for linear and non-linear composites with arbitrary phase contrast. International Journal for Numerical Methods in Engineering 52(1): 139-160.

Monchiet V and Bonnet G (2012) A polarization-based fft iterative scheme for computing the effective properties of elastic composites with arbitrary contrast. International Journal for Numerical Methods in Engineering 89(11): 1419-1436.

Monchiet V and Kondo D (2012) Exact solution of a plastic hollow sphere with a mises-schleicher matrix. International Journal of Engineering Sciences 51: 168-178.

Monchiet V, Cazacu O, Charkaluk E, et al. (2008) Macroscopic yield criteria for plastic anisotropic materials containing spheroidal voids. International Journal of Plasticity 24(7): 1158-1189.

Monchiet V, Charkaluk E and Kondo D (2014) Macroscopic yield criteria for ductile materials containing spheroidal voids: An eshelby-like velocity fields approach. Mechanics of Materials 72: 1-18.

Morin L, Leblond JB, Benzergaand AA, et al. (2016) A unified criterion for the growth and coalescence of microvoids. Journal of the Mechanics and Physics of Solids 97: 19-36.

Morin L, Leblond JB and Kondo D (2015) A gurson-type criterion for plastically anisotropic solids containing arbitrary ellipsoidal voids. International Journal of Solids and Structures 77: 86-101.

Moulinec H and Suquet P (1994) A fast numerical method for computing the linear and nonlinear mechanical properties of composites. Comptes rendus de l'academie des sciences t.318, Serie II: 1417-1423.

Moulinec H and Suquet P (1998) A numerical method for computing the overall response of nonlinear composites with complex microstructure. Computer Methods in Applied Mechanics and Engineering 157(1): 69-94.

Pardoen T and Hutchinson J (2003) Micromechanics-based model for trends in toughness of ductile metals. Acta Materialia 51(1): 133-148.

Ponte Castaneda P (1991) The effective mechanical properties of nonlinear isotropic composites. Journal of the Mechanics and Physics of Solids 39(1): 45-71.

Shen W, He Z, Dormieux L, et al. (2014a) Effective strength of saturated double porous media with a druckerprager solid phase. International journal for numerical and analytical methods in geomechanics 38(3): 281-296. 
Shen W, Lanoye E, Dormieux L, et al. (2014b) Homogenization of saturated double porous media with eshelby-like velocity field. Acta Geophysica 62(5): 1146-1162.

Shen W, Oueslati A and De Saxce G (2015a) Macroscopic criterion for ductile porous materials based on a statically admissible microscopic stress field. International Journal of Plasticity 70: 60-76.

Shen W, Pastor F and Kondo D (2013) Improved criteria for ductile porous materials having a green type matrix by using eshelby-like velocity fields. Theoretical and Applied Fracture Mechanics 67-68: 14-21.

Shen W, Shao J, Kondo D, et al. (2015b) A new macroscopic criterion of porous materials with a MisesSchleicher compressible matrix. European Journal of Mechanics A/Solids 49: 531-538.

Shen WQ, Lin J, Zhu QZ, et al. (2011) Macroscopic yield criterion for ductile materials containing randomly oriented spheroidal cavities. International Journal of Damage Mechanics 20(8): 1198-1216.

Shen WQ, Shao JF, Dormieux L, et al. (2012) Approximate criteria for ductile porous materials having a green type matrix: Application to double porous media. Computational Materials Science 62: 189-194.

Sun Y and Wang D (1989) A lower bound approach to the yield loci of porous materials. Acta Mechanica 5(3): 237-243.

Tvergaard V (1981) Influence of voids on shear bands instabilities under plane strain conditions. International Journal of Fracture 17(4): 389-407.

Tvergaard V (1982) Material failure by void coalescence in localized shear bands. International Journal of Solids and Structures 18(8): 659-672. 